\title{
Biomarkers, myocardial fibrosis and co-morbidities in heart failure with preserved ejection fraction: an overview
}

\author{
Marta Michalska-Kasiczak ${ }^{1,2}$, Agata Bielecka-Dabrowa ${ }^{1,3}$, Stephan von Haehling ${ }^{4}$ \\ Stefan D. Anker ${ }^{5,6}$, Jacek Rysz ${ }^{7}$, Maciej Banach ${ }^{1}$
}

\author{
'Department of Hypertension, Chair of Nephrology and Hypertension, Medical \\ University of Lodz, Lodz, Poland \\ ${ }^{2}$ Department of Endocrine Disorders and Bone Metabolism, $1^{\text {st }}$ Chair of Endocrinology, \\ Medical University of Lodz, Lodz, Poland \\ ${ }^{3}$ Department of Cardiology and Congenital Diseases of Adults, Polish Mother's \\ Memorial Hospital Research Institute, Lodz, Poland \\ ${ }^{4}$ Department of Cardiology and Pneumology, University of Göttingen Medical Center, \\ Göttingen, Germany \\ ${ }^{5}$ Division of Cardiology and Metabolism - Heart Failure, Cachexia and Sarcopenia, \\ Department of Cardiology, Campus Virchow-Klinikum, Charité - Universitätsmedizin \\ Berlin, Berlin, Germany \\ ${ }^{6}$ Berlin-Brandenburg Center for Regenerative Therapies (BCRT), Charité- \\ Universitätsmedizin Berlin, Berlin, Germany \\ 'Department of Nephrology, Hypertension and Family Medicine, Chair of Nephrology \\ and Hypertension, Medical University of Lodz, Lodz, Poland
}

Submitted: 17 April 2018

Accepted: 24 April 2018

Arch Med Sci 2018; 14, 4: 890-909

DOI: https://doi.org/10.5114/aoms.2018.76279

Copyright (c) 2018 Termedia \& Banach

\section{Abstract}

The prevalence of heart failure with preserved ejection fraction (HFpEF) is steadily increasing. Its diagnosis remains difficult and controversial and relies mostly on non-invasive echocardiographic detection of left ventricular diastolic dysfunction and elevated filling pressures. The large phenotypic heterogeneity of HFpEF from pathophysiological underpinnings to clinical manifestations presents a major obstacle to the development of new therapies targeted towards specific HF phenotypes. Recent studies suggest that natriuretic peptides have the potential to improve the diagnosis of early HFpEF, but they still have significant limitations, and the cut-off points for diagnosis and prognosis in HFpEF remain open to debate. The purpose of this review is to present potential targets of intervention in patients with HFpEF, starting with myocardial fibrosis and methods of its detection. In addition, co-morbidities are discussed as a means to treat HFpEF according to cut-points of biomarkers that are different from usual. Biomarkers and approaches to co-morbidities may be able to tailor therapies according to patients' pathophysiological needs. Recently, soluble source of tumorigenicity 2 (SST2), growth differentiation factor 15 (GDF-15), galectin-3, and other cardiac markers have emerged, but evidence from large cohorts is still lacking. Furthermore, the field of miRNA is a very promising area of research, and further exploration of miRNA may offer diagnostic and prognostic applications and insight into the pathology, pointing to new phenotype-specific therapeutic targets.

Key words: biomarkers, heart failure with preserved ejection fraction, microRNA, diagnosis.

\section{Corresponding authors:}

Agata Bielecka-Dabrowa MD, PhD, Assoc Prof.

Prof. Maciej Banach MD, PhD Department

of Hypertension Chair of Nephrology and Hypertension Medical University of Lodz

113 Zeromskiego St 90-549 Lodz, Poland Phone/fax: +48 426393771 E-mail: agatbiel7@poczta. onet.pl, maciejbanach@aol.co.uk 


\section{Introduction}

\section{Classification of heart failure}

Heart failure (HF) is a clinical syndrome characterized by substantial prevalence and mortality, frequent hospitalizations, poor quality of life, and complex treatment regimens [1]. Two main types of $\mathrm{HF}$ are differentiated: HF with reduced ejection fraction (HFrEF), also known as systolic HF; and $\mathrm{HF}$ with preserved ejection fraction (HFpEF), also known as diastolic HF $[2,3]$. Very recently, the European Society of Cardiology (ESC) recommended describing a third type: HF with medium-range ejection fraction (HFmrEF) [4].

The guidelines of the ESC recommend basing the diagnosis of HFpEF on typical symptoms and signs of HF (volume overload, dyspnoea, exercise intolerance) and a left ventricular ejection fraction (LVEF) of more than $50 \%$. More specific diagnostic criteria have evolved over time and include signs and symptoms of HF, objective evidence of diastolic dysfunction (impaired left ventricular (LV) relaxation or increased LV diastolic stiffness), disturbed LV filling, structural heart disease, and elevated B-type natriuretic peptide (BNP) or its precursor N-terminal pro-BNP [5-7]. However, it may happen that HFpEF is mimicked by restrictive cardiomyopathy, dynamic mitral regurgitation, ischaemic heart disease, pericardial disease and exercise-induced pulmonary hypertension [8]. Even though the ESC has suggested diagnostic criteria of HFpEF, enrolment criteria for HFpEF trials have varied considerably over the last decade (Table I) [9-14].

It is estimated that about half of all patients presenting with signs and symptoms of HF have HFpEF. In the past decade, the relative proportion of patients with the diagnosis of HFpEF compared with those with HFrEF has increased, suggesting the increasing importance of the syndrome, but also increasing awareness of its presence [15-18].

The aim of this review is to provide an overview of biomarkers whose assessment may be useful in patients with HFpEF, taking the pathophysiology of inflammatory mechanisms and myocardial fibrosis into consideration as well as patients' co-morbidities. Since a "one-size fits all" approach to HFpEF has proven unsuccessful in the past, the development of treatment strategies that tailor pharmacological intervention to pathophysiological needs is very promising. Biomarkers may be extremely useful tools in this undertaking.

\section{Pathophysiology of HFpEF}

Heart failure with preserved ejection fraction is a syndrome with a number of underlying aetiologies and numerous pathophysiological alterations contributing to the heterogeneous disease state
[19-21]. Myocardial remodelling and dysfunction in HFpEF include myocardial hypertrophy as well as progressive myocardial fibrosis and increased cardiomyocyte stiffness. These characteristics result from specific changes in transcriptional and post-translational modifications of a huge, elastic sarcomeric protein named titin [22-26]. Extracellular matrix accumulation and fibrosis are often described, and may significantly contribute to impaired LV filling, a hallmark of the disease [27]. Fibrosis is the result of an imbalance between exaggerated collagen synthesis and unchanged or depressed collagen degradation. As compared to HFrEF, patients with HFpEF are generally older, more often female and have a high prevalence of non-cardiac and cardiac co-morbidities such as hypertension, overweight/obesity, diabetes mellitus, chronic obstructive pulmonary disease, atrial fibrillation, anaemia, or chronic kidney disease even after leaving aside specific causes of HF in the context of a normal or near normal LVEF (e.g., hypertrophic cardiomyopathy, cardiac amyloidosis, Fabry disease) [28-30]. Recently, Paulus et al. [31] postulated a novel paradigm, which identifies a systemic pro-inflammatory state induced by co-morbidities as the origin of microvascular endothelial cell inflammation. Inflammation in response to co-morbid conditions may play a proportionally larger role in HFpEF than in HFrEF [31]. On the other hand, haemodynamic overload evidenced by increased LV diastolic pressure (LVDP) and myocardial fibrosis evidenced by increased extracellular matrix fibrillar collagen may be important mechanisms contributing to the development of HFpEF [32]. For example, Schelbert et al. investigated whether myocardial fibrosis is similarly prevalent in those with HFpEF and those at risk of HFpEF, and whether it is similarly associated with disease severity and outcomes [33]. Of 1174 patients identified, 250 were "at risk" of HFpEF given an elevated BNP level; 160 had HFpEF as a documented clinical diagnosis, and the remaining 745 did not have either. Patients at risk of HFpEF or with HFpEF demonstrated similarly higher prevalence/extent of myocardial fibrosis and worse prognosis compared with patients with no HFpEF. Over a median of 1.9 years of follow-up, 61 patients at risk of HFpEF or with manifest HFpEF experienced adverse events (19 hospitalizations for HF, 48 deaths, 6 with both). Regardless, myocardial fibrosis was associated with disease severity (i.e., BNP) and outcomes. Conceivably, myocardial fibrosis may precede a clinical HFpEF diagnosis [33]. Unfortunately, the measurement of LVDP and fibrosis generally requires either invasive procedures and/or complex and sophisticated imaging techniques. However, biomarkers measured in the plasma have been shown to ac- 


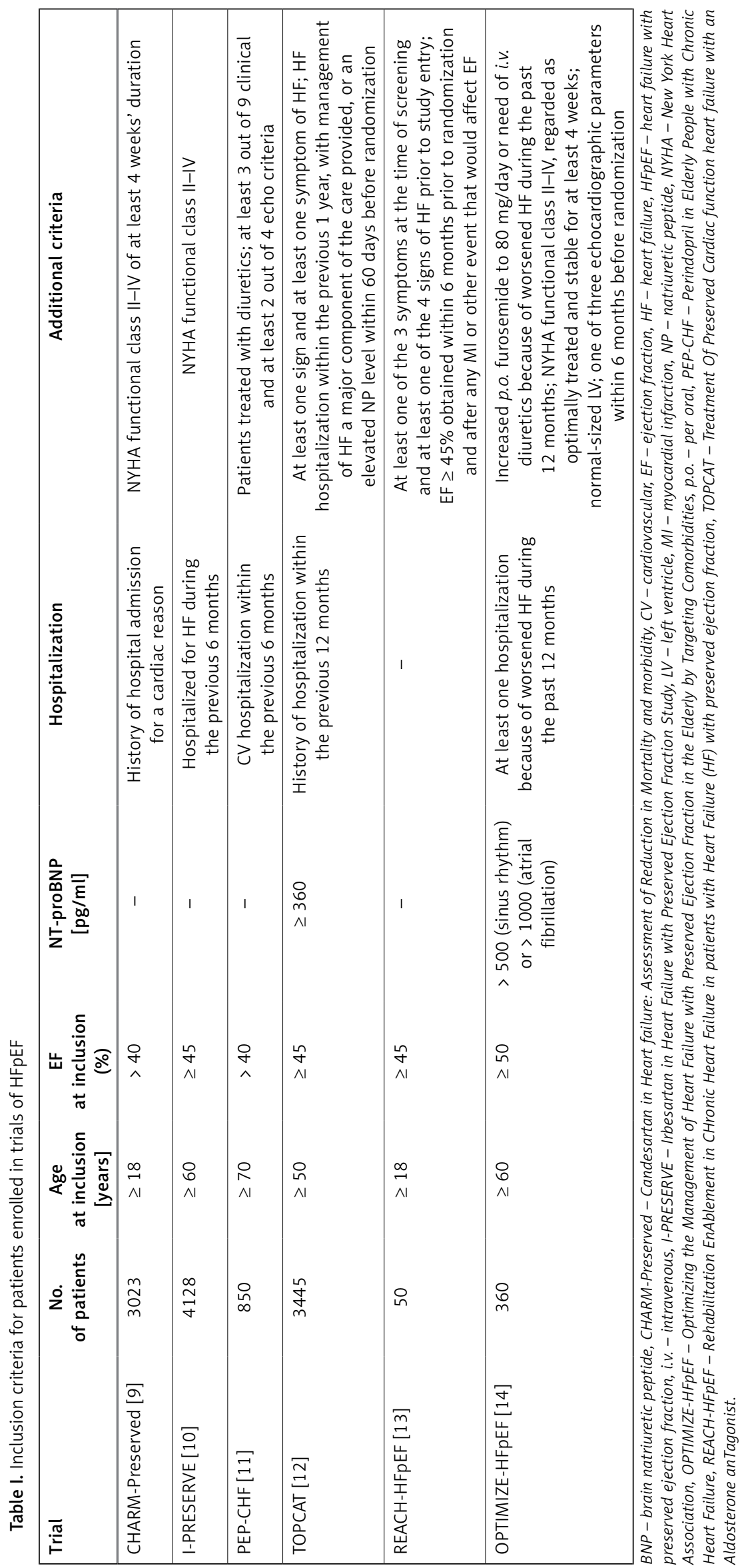


curately reflect changes in haemodynamic load and myocardial fibrosis and may have important applications to the management of patients with HFpEF [34]. For example, matrix metalloproteinases (MMPs), tissue inhibitors of MMPs (TIMPs), and the collagen processing proteins collagen propeptides and collagen telopeptides reflect changes in collagen homeostasis and the transition from antecedent diseases such as hypertension to clinically symptomatic HFpEF [34, 35]. Galectin-3 and soluble source of tumorigenicity 2 (sST2) reflect the general extent of fibrosis and the severity of HFpEF, and they are also good candidates for determining the early stage of HF development influenced fibrosis. Apart from the aforementioned markers, growth differentiation factor 15 (GDF-15) and microRNAs (miRNA) are promising new biomarkers for the detection of fibrosis [33-35].

\section{Biomarkers in HFpEF}

The diagnosis of HFpEF remains challenging and so far largely relies on echocardiography and natriuretic peptide testing. Additional indicators of HFpEF including biomarkers that reflect left ventricular filling pressure (LVFP) would be of great clinical value. Biomarkers in this context embrace parameters measured in blood that may provide important information on the pathogenesis of HFpEF, but may also be a valuable clinical tool in the identification of patients at risk for $\mathrm{HFpEF}$, in the diagnosis of HFpEF, in risk stratification, and in monitoring therapy. However, most of the known biomarkers are poorly validated for their use in clinical trials, and there is a need for new biochemical and imaging parameters enabling physicians to assign cardiac dysfunction to the symptoms of the patient [31]. Currently we are awaiting the results of a study which aims to identify new biomarkers of HFpEF progression and to find pathophysiological mechanisms to support explorations of new treatment regimens for HFpEF [36]. The result may not be a single biomarker, but rather a panel of biomarkers that identifies different pathophysiological changes, as has been used in previous studies [37]. Indeed, the Preserved and Reduced Ejection Fraction Epidemiological Regional Study (PREFERS) Stockholm HF study will enable the characterization and comparison of new onset HFpEF and HFrEF patients by using new cutting edge techniques such as advanced bioinformatics and spatial transcriptomics [36]. Our selection of biomarkers was based on our own investigations and research experience $[27,38,39]$.

\section{Search strategy}

We searched the electronic databases MEDLINE (1966 to January 2018), EMBASE and SCOPUS (1965 to January 2018). Additionally, abstracts from national and international cardiovascular meetings were searched. When necessary, the relevant authors were contacted to obtain further data. The main data search terms were: heart failure with preserved ejection fraction, diastolic heart failure, heart failure with normal ejection fraction, biomarker(s), marker(s), microRNA, diagnosis and heart failure, comorbidities and heart failure.

\section{Natriuretic peptides as the gold standard}

The biologically active BNP and its inactive amino-terminal fragment NT-proBNP are synthesized within the ventricular myocardium in response to myocyte stretch and/or pressure overload. The major physiological effects of BNP are natriuresis, vasodilation and inhibition of the renin-angiotensin-aldosterone system (RAAS) and the sympathetic nervous system [40, 41]. BNP is quickly degraded by endopeptidases and natriuretic peptide receptor C (NPR-C) and has a half-life of approximately 20 min. By contrast, NT-proBNP circulates as a biologically inert substance that is cleared passively primarily by the kidney, and accordingly has a longer half-life of approximately 1 to 2 h [40, 41].

Recent studies have shown that the plasma levels of BNP and NT-proBNP are powerful biomarkers for the diagnosis and prognosis of patients with HF. However, there are also limitations to natriuretic peptide use that need to be kept in mind when interpreting their results. Apart from advancing age and ventricular function, other factors influence the clinical interpretation of BNP and NT-proBNP, including obesity, renal dysfunction, atrial arrhythmias, and cardiotoxic agents as well as structural heart disease [42]. The diagnostic cut-off values to rule out HF suggested by the ESC, however, apply to both HFrEF and HFpEF. For BNP, a cut-off value of $<35 \mathrm{pg} / \mathrm{ml}$ has been suggested, while for NT-proBNP the corresponding value is $<125 \mathrm{pg} / \mathrm{ml}$ even though patients older than 75 years may benefit from using a threshold of $<400 \mathrm{pg} / \mathrm{ml}$. Despite the fact that circulating levels of natriuretic peptides are elevated in patients with HFpEF as compared to subjects without HF, they are usually lower than levels seen in patients with HFrEF [43]. Sanders-van Wijk et al. [44] supported these findings with regard to differential expression of NT-proBNP in HFpEF compared with HFrEF. HFrEF patients exhibited higher NT-proBNP values than those with HFpEF (4202 pg/ml (interquartile range (IQR): 2239-7411, pg/ml) vs. 2142 $\mathrm{pg} / \mathrm{ml}$ (IQR: 1473-4294 pg/ml) vs. $p<0.001$ ). Moreover, it was found that NT-proBNP had significantly lower prognostic value in HFpEF compared with HFrEF in the NT-proBNP-guided study arm only [44]. However, in another study the authors found that, upon reaching a certain concentration, 
the prognostic value of BNP was not different in HFpEF vs. HFrEF $[45,46]$. Because HFpEF patients usually have a smaller LV cavity and thicker LV walls, their end diastolic wall stress is much lower than in HFrEF, even in the setting of high diastolic pressures, thus producing a lower stimulus for BNP production [47]. In cases where BNP/NT-pro$B N P$ is required to be elevated for a definite diagnosis of HF, only the higher risk HFpEF patients will be identified, resulting in a reduced prevalence of HFpEF, especially when current guidelines [4] recommend the same cut-off points for NT-proBNP and BNP levels for all kinds of HF. The above considerations suggest that natriuretic peptides may be somewhat less useful as a diagnostic and prognostic tool in HFpEF as compared to HFrEF [48]. On the other hand, Jhund et al. [49] studied more than 4,000 patients from the I-Preserve trial aged $\geq 60$ years in New York Heart Association (NYHA) class II-IV HF with LVEF $\geq 45 \%$. They found that beyond NT-proBNP increases or decreases of 1000 $\mathrm{pg} / \mathrm{ml}$ from baseline, there was little change in the risk of cardiovascular death or HF hospitalization. This study therefore suggests that the value of NT-proBNP, which can help the clinician to monitor prognosis, is higher than might be expected.

In the study of van Veldhuisen et al. [45] BNP levels were overall lower in patients with HFpEF than in those with HFrEF, but the associated risk for reaching the primary endpoint was at least similar for a given BNP level. These findings indicated, however, that for a given BNP level, which would suggest a similar severity of HF, the associated risk is the same for HFpEF and HFrEF, and this was true for patients with low, intermediate, and high BNP levels [45]. The applicability of only natriuretic peptides for the entire spectrum of $\mathrm{HF}$ is difficult, because there are still questions about the cut-off levels to recognize and monitor HFpEF. This might be important information for future HFpEF trials and strengthens the fact that the sole use of natriuretic peptides as the only HF markers may be insufficient [50].

The concentration of atrial natriuretic peptide (ANP) in the circulation is approximately 10- to 50fold higher than that of BNP, and its production is increased in response to increased atrial wall stretch in HF [51]. However, reliable detection of circulating ANP concentrations can be challenging because its half-life is only $2-5 \mathrm{~min}$ [52]. The 126 amino acid prohormone of ANP, designated as proANP, has a longer half-life and makes serum measurement more feasible [42]. A novel immunoassay that detects the mid-regional sequence of proANP (MR-proANP) is now available, and has been evaluated as a diagnostic and prognostic test for HF patients [53]. Recently, Andersen et al. [54] found in subjects recovering from an acute myocardial infarction with preserved LVEF a significant correlation between MR-proANP (but not NT-proBNP) and pulmonary capillary wedge pressure at rest $(p=0.002)$ and at peak exercise $(p=0.002)$. MR-proANP was measured at rest before the exercise.

In conclusion, when using natriuretic peptides for making a diagnosis of HFpEF, their plasma levels should not be used in isolation from the clinical context and echocardiography. Because natriuretic peptides are influenced by many factors other than diastolic function, their measurements may give false positive results for HF. On the other hand, the relatively low natriuretic peptide levels suggest lower diastolic wall stress in HFpEF when compared to HFrEF, which may give false negative results. In this context, besides natriuretic peptides, additional biomarkers are needed to characterize HFpEF in more detail.

\section{Biomarkers of fibrosis in HFpEF}

\section{Soluble source of tumorigenicity 2 (sST2)}

Source of tumorigenicity 2 (ST2) is a receptor belonging to the interleukin-1 receptor family that is expressed in cardiomyocytes and elsewhere [55]. The receptor has 2 isoforms: a transmembrane form and a soluble circulating form (sST2), which lacks the intracellular and transmembrane domains. The ligand for both isoforms is the cytokine interleukin-33 (IL-33), which, when bound to the transmembrane form of the receptor, exerts a potentially protective antihypertrophic, antifibrotic effect on the cardiomyocyte [56, 57]. sST2 binds and removes IL-33 from the circulation, thus potentially promoting cardiac hypertrophy, fibrosis, and ventricular dysfunction. SST2 has been found to have diagnostic and prognostic value in patients with destabilized HF, acute coronary syndrome [58] and advanced chronic HF, and was included in the 2013 ACCF/AHA guidelines for additive risk stratification of patients with acute and chronic HF [59]. However, the diagnostic utility of sST2 in patients with HFpEF has been less well studied. A post-hoc analysis of 200 patients with HFpEF provided the first evidence that SST2 levels could be of interest in patients with HFpEF [55]. In the subpopulation of individuals with normal systolic function and acute dyspnoea, only SST2 continued to predict mortality. The authors found an approximately 2-fold higher median SST2 concentration in those with an E/e' ratio > 15 (suggestive of increased LV end-diastolic filling pressure) than in the remaining patients, but, in contrast, the median NT-proBNP was approximately 157fold higher in those with an $E / e^{\prime}$ ratio $>15$ than in the others. The weak correlation of SST2 with echocardiographic parameters in this unselect- 
ed dyspnoea population, compared with NT-proBNP, calls into question the clinical relevance of the stretch-mediated pathway of SST2 secretion. The results of studies assessing the significance of the concentration of SST2 in patients with HFpEF are likewise inconclusive. Santhanakrishnan et al. found that HFpEF patients had higher serum levels of SST2 compared with healthy subjects, but healthy subjects per se may not be the ideal comparator [60]. Besides, this difference did not remain statistically significant after adjustment for age, sex, and clinical covariates. Wang et al. [61] evaluated 107 hypertensive patients with LVEF > 50\%. Among them, 68 presented with symptoms of HFpEF. The area under the receiver-operator curve (AUC) was 0.80 for SST2 as compared to 0.70 for NT-proBNP to correctly diagnose HFpEF. The SST2 concentration was significantly lower in patients with $E / e<8$ compared with those with $E / e^{\prime} 8-15$ or $E / e^{\prime}>15$. Multivariate analysis demonstrated that SST2 $>13.5 \mathrm{ng} / \mathrm{ml}$ was independently associated with the presence of HFpEF. Jhund et al. [62] studied 296 patients from the Prospective Comparison of Angiotensin Receptor-Neprilysin Inhibitors (ARNI) with Angiotensin II receptor blocker (ARB) on Management of Heart Failure With Preserved Ejection Fraction (PARAMOUNT) trial with a measurement of sST2. Higher SST2 was associated with older age, male sex, atrial fibrillation, higher NYHA class, higher NT-proBNP and lower estimated glomerular filtration rate (eGFR). Increasing SST2 levels were associated with higher $E / e^{\prime}$ and larger left atrial size. Associations were similar after excluding patients with atrial fibrillation. In a multivariable model, male sex, increasing NYHA class and left atrial volume were independently associated with higher sST2 [62].

More clinical data on sST2 in HFpEF were provided by Friões et al. [63] In this study, the authors divided patients with acute HF according to their LVEF, and reported that NT-proBNP predicted allcause mortality or HF readmission at 6 months regardless of LVEF. SST2 was found to be a significant predictor of prognosis in HFrEF, but not in HFpEF patients. By contrast, in another analysis the authors demonstrated comparable prognostic value of SST2 in both HFpEF and HFrEF patients [64]. Hypertension is the most prevalent co-morbidity in patients with HFpEF and might lead to activation of different pathophysiological mechanisms in the course of the disease. This has been recently investigated by Zile et al. [24] SST2 was measured in 70 coronary artery bypass grafting (CABG) patients stratified into three groups: control (no hypertension), hypertensive patients without HFpEF, and hypertensive patients with HFpEF. The authors observed that SST2 was higher in hypertensive patients without HFpEF com- pared with the control subjects. Moreover, the SST2 levels were raised in hypertensive patients with HFpEF $(105.5 \pm 31.4$ vs. $82.0 \pm 35.7 \mathrm{ng} / \mathrm{ml}$; $p$ <0.05) [24]. In the study of Parikh et al. [65] in 3915 older, community-dwelling subjects from the Cardiovascular Health Study without prevalent HF, sST2 level above the US Food and Drug Administration-approved cut-off value (>35 $\mathrm{ng} / \mathrm{ml}$ ) was significantly associated with incident HF (hazard ratio (HR) 1.20; 95\% confidence interval $(\mathrm{Cl})$ : 1.02-1.43) and cardiovascular death $(H R=1.21$; $95 \% \mathrm{Cl}: 1.02-1.44)$, and greater SST2 was continuously associated with cardiovascular death (per 1-In increment: $\mathrm{HR}=1.24 ; 95 \% \mathrm{Cl}: 1.02-1.50)$. Addition of SST2 to existing risk models of HF and cardiovascular death modestly improved discrimination and reclassification into a higher risk [65].

Summarizing research on the importance of SST2 concentrations in HFpEF, the ambiguity of the results draws our attention, and available studies remain retrospective in nature and altogether rather inconclusive. One of the important points in this regard is the heterogeneity of inclusion criteria used across different studies, rendering even meta-analyses difficult. SST2 could be used as a biomarker to predict HFpEF. However, such an approach may still merit consideration in order to provide additional insight.

\section{Growth differentiation factor 15}

Growth differentiation factor 15 (GDF-15) is a member of the transforming growth factor- $\beta$ cytokine superfamily and a marker of cell injury and inflammation. GDF-15 could be used as a novel biomarker to evaluate myocardial fibrosis. In the study by Zhou et al. [66] after linear correlation analysis, the expression GDF-15 was found to be positively related to the degree of cardiac fibrosis in patients with atrial fibrillation [66]. In patients with end-stage non-ischemic dilated cardiomyopathy, serum GDF-15 level correlated with the severity of myocardial fibrosis [67]. Expression of GDF-15 is strongly induced in cardiomyocytes in response to metabolic stress such as cardiac ischaemia (nitric oxide-dependent), tissue injury, or a pressure overload state (angiotensin 2-dependent) [42, 68, 69]. Serum GDF-15 levels positively correlate with LV mass in elderly individuals [70], and a high level of plasma GDF-15 is an independent predictor of LV hypertrophy in patients with hypertension [71]. It was recently found that circulating GDF-15 is a useful biomarker for differentiating hypertrophic cardiomyopathy from hypertensive LV hypertrophy [72]. Izumiya et al. [73] reported that serum GDF-15 levels were positively correlated with NYHA functional class $(p \leq 0.0001)$, plasma BNP levels $(p \leq 0.0001)$, and serum high sensitive troponin T levels $(p=0.001)$ in 
150 patients with LV diastolic dysfunction (LVDD) and with HFpEF (LVEF > 50\%). Serum GDF-15 levels in the HFpEF group were significantly higher than those in the LVDD group $(p<0.0001)$. In another small population study $(n=151)$, the discriminatory capacity of GDF-15 (area under the receiver-operator curve $(\mathrm{AUC})=0.936)$ was similar to that of NT-proBNP (AUC $=0.934$ ) for HFpEF versus controls [60]. Increasing levels of both biomarkers correlated with worsening diastolic indices. The ratio NT-proBNP : GDF-15 best distinguished HFpEF from HFrEF (AUC = 0.709) [60].

Sinning et al. [74] recently described GDF-15 as a useful marker to detect prevalent HF in addition to NT-proBNP and found elevated levels in HFrEF and HFpEF, whereas NT-proBNP was higher in HFrEF than in HFpEF. All biomarkers were useful to predict mortality in the general population. The index calculated from the equation ((C-reactive protein [CRP]+GDF-15+sST2)/NT-proBNP) was able to discriminate HFpEF from HFrEF [74]. The prognostic value of GDF-15 was also confirmed in recent studies. GDF-15 remained a significant independent predictor of the composite outcome even after adjusting for important clinical predictors including high-sensitivity troponin $\mathrm{T}$ and NT-proBNP (adjusted hazard ratio 1.76 per $1 \mathrm{Ln} \mathrm{U}$, 95\% Cl: $1.39-2.21 ; p<0.001)$, regardless of $\mathrm{HF}$ group ( $p$-value for interaction $=0.275)[75,76]$ The study by Kopytsya et al. [77] detected an association between high levels of GDF-15, determined within the first $24 \mathrm{~h}$ after acute coronary syndrome, and congestive HF progression after 12 months. Overall, GDF-15 could serve as a biomarker helpful in differentiating the type of HF preserved vs. reduced EF - and additionally as an indicator of prognosis.

\section{Galectin-3}

Myocardial fibrosis resulting in increased myocardial stiffness is a major component of HFpEF pathophysiology, and it is a manifestation of collagen synthesis (or decreased collagen degradation), inflammation, and oxidative stress [76, 78-80]. Galectin-3 is a macrophage product and member of the family of soluble $\beta$-galactoside-binding lectins. It can be found on a wide variety of cells and tissue surfaces. The function of galectin-3 is probably related to the inflammatory cascade following cardiac injury, as well as pathways regulating cardiac contractility [79]. Recent studies have confirmed that galectin-3 is linked to HF development and that it is implicated in a variety of processes [80] that are thought to play roles in the pathophysiology of HFpEF including myofibroblast proliferation, fibrogenesis, tissue repair, inflammation, and ventricular remodelling [81-83]. In the study of Wu et al. [84], both plasma and myocar- dial galectin-3 levels correlated with the severity of diastolic dysfunction. To date, only a few clinical studies have described the clinical value of galectin-3 in patients with HFpEF. The first study to identify galectin-3 as a predictive marker for $\mathrm{HF}$ was a substudy from the Pro-BNP Investigation of Dyspnea in the Emergency Department (PRIDE) study [85]. In a subanalysis of about 200 patients diagnosed with acute decompensation of $\mathrm{HF}$, galectin-3 levels were correlated with echocardiographic measures of higher filling pressure (higher E/e' ratio), abnormalities of diastolic relaxation (lower $e^{\prime}$ velocity), and valvular regurgitation. Additionally, the highest levels of galectin-3 were associated with a higher risk of 4-year mortality, independent of LV dimensions and function [86]. In the Advising and Counseling in Heart Failure $(\mathrm{COACH})$ trial, galectin-3 was found to be an independent predictor of all-cause mortality or HF hospitalization during 18 months of follow-up in 592 patients with NYHA class II-IV HF (hazard ratio (HR) 1.38, 95\% confidence interval (CI): 1.07-1.78, $p=0.015$; adjusted for age, gender, BNP, eGFR, and diabetes) [87]. De Boer et al. [87] observed that increasing quartiles of baseline galectin-3 levels were associated with a greater risk for all-cause mortality or HF hospitalization in patients with HFpEF (defined as LVEF > 40\%) than in patients with HFrEF $(n=485)$ [87]. Similar results were obtained in another study in which galectin-3 above the median of $13.8 \mathrm{ng} / \mathrm{ml}$ independently predicted all-cause mortality of 419 patients hospitalized with HF and an LVEF > 45\% [88].

Currently, there are few data regarding the relationship between galectin-3 levels and the response to specific pharmacological or non-pharmacological therapeutic approaches in HFpEF. This is in contrast to data available in cohorts of patients with HFrEF, where there is some evidence that baseline galectin-3 levels are predictive for the response to a specific drug therapy $[89,90]$. For example, in the Aldosterone Receptor Blockade in Diastolic Heart Failure (Aldo-DHF) trial [91], the authors determined the association between galectin-3 levels and patient characteristics in HFpEF and assessed the association between galectin-3 and clinical outcomes. The study was performed in patients in NYHA classes II-III, $\operatorname{LVEF} \geq 50 \%$, grade $\geq$ I diastolic dysfunction, and peak oxygen consumption (peakVO ${ }_{2}$ ) $\leq 25 \mathrm{ml} / \mathrm{kg}$ / min. Following multivariable adjustment, baseline galectin-3 inversely correlated with peak $\mathrm{VO}_{2}$, 6 min walk distance, and Short Form 36 (SF-36) physical functioning and with NYHA class. At 6 and 12 weeks, increasing galectin-3 levels were associated with all-cause death or hospitalization independent of treatment arm ( $H R=3.319$, 95\% Cl: 1.214-9.07, $p=0.019)$ and NT-proBNP $(\mathrm{HR}=3.127,95 \% \mathrm{Cl}: 1.144-8.549, p=0.026)$ [91]. 
AbouEzzeddine et al. [92] observed that in overt HFpEF galectin-3 was related to severity of renal dysfunction and, accounting for this, it was not independently associated with the severity of pathophysiological derangements. These findings underscore the need to adjust for renal function when interpreting galectin-3 levels, and call into question the value of galectin-3 in quantifying disease severity in overt HFpEF [92]. Altogether, galectin-3, like sST2, is an interesting candidate biomarker that may find a role in HFpEF. However, in the absence of clinical studies and clinical algorithms that establish the value of specific cut-offs, their clinical importance remains elusive.

\section{Circulating microRNAs}

MicroRNAs (miRNAs) are small (17-25 nucleotides) non-coding RNAs that play a role in gene expression by binding to the $3^{\prime}$-untranslated regions of target messenger RNAs (mRNAs). miRNAs initiate either translational repression or degradation of mRNAs, thereby regulating gene expression at the post-translational stage (Figure 1) [93-95]. They are found in humans, animals, plants, and viruses [96]. Due to the ability of a single miRNA to target hundreds of mRNAs and their involvement in biological processes including stem cell differentiation, phase change, signalling, and response to stress, incorrect miRNA expression may be associated with the development of $\mathrm{HF}$ and is being viewed as a potential biomarker of this disease [97].

Identification of aberrant miRNA expression in the plasma could be useful in early and differential diagnosis of selected diseases. In this context, the recent detection of miRNAs in various body fluids (e.g. blood, saliva, serum, milk) has led researchers to consider them as intriguing candidate biomarkers for various diseases [97]. MicroRNAs offer many attractive features as biomarkers. They are stable in the circulation, their sequences are evolutionarily conserved, their expression is often tissue or pathology specific, and their detection is based on sequence-specific amplification - requisite features for valuable biomarkers [98]. The diagnostic potential of miRNA detection in human

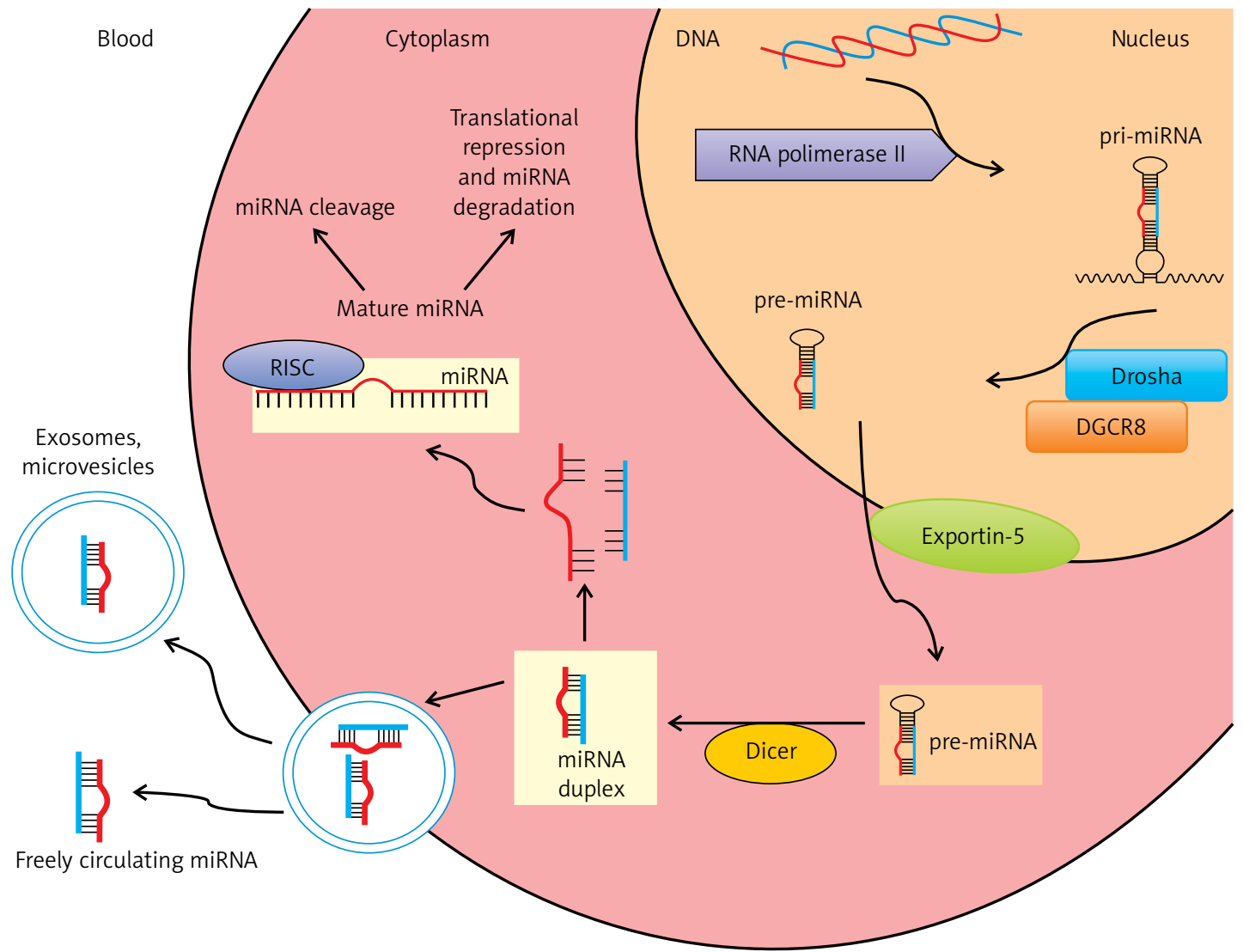

Figure 1. MicroRNA biogenesis pathway. In the nucleus, miRNAs are transcribed by RNA polymerase II to generate long primary transcripts (pri-miRNAs) which may contain more than one miRNA. Pri-miRNAs are subsequently processed by the endonuclease Drosha and its binding partner DGCR8, forming hairpin-like precursor miRNAs (pre-miRNAs). Pre-miRNAs are exported into the cytoplasm by exportin-5. Pre-miRNAs are cleaved by the RNase III enzyme (Dicer) to mature miRNAs. The mature miRNAs are incorporated into the RNA-induced silencing complex (RISC), in this form leading to degradation of target miRNAs and/or inhibition of translation. Mature miRNAs can be released into the circulation, incorporated into vesicles such as multivesicular bodies (MVB), exosomes, microvesicles or as freely circulating miRNAs 
plasma for cardiovascular disorders is beginning to be explored. Recent studies have shown that miRNAs are also associated with cardiac disease and $\mathrm{HF}$, and their expression in myocardial tissue was more sensitive than mRNAs for the prediction of functional status in HF patients [99]. Thus far, distinctive patterns of circulating miRNAs have been found for myocardial infarction, HF, atherosclerosis, type 2 diabetes, and hypertension. Although these reports indicate that plasma levels of specific miRNAs correspond to different forms of cardiovascular disease, they do not currently determine whether plasma miRNA levels correspond to a deterioration or improvement in heart disease in humans [100].

It was recently reported that miRNAs, which can be reliably isolated from human serum and are stable in the circulation, can be used as a diagnostic tool for early HF detection [101]. One study has shown that miR-1246 and miR-124-5p in the buffy coat might be used as biomarkers for the assessment of diastolic dysfunction and dilated cardiomyopathy [102]. The authors suggest that in patients with diastolic dysfunction with or without systolic dysfunction, there is striking dysregulation of plasma levels of miR-454, miR-142-3p and miR-500. Conversely, miR-1246 was steadily upregulated in all three groups (DD - isolated diastolic dysfunction; DCM - stable compensated dilated cardiomyopathy; DCM-CHF - decompensated congestive heart failure secondary to dilated cardiomyopathy), while miR-124-5p was significantly up-regulated in the DCM group. In patients with isolated diastolic dysfunction, the expression of miR-1246 levels demonstrated highly specific diagnostic accuracy, while miR-124-5p showed highly specific diagnostic accuracy for DCM prediction. These findings point to the possibility of a functional correlation between dysregulation of these miRNAs and target gene alteration in the distant organ. Nevertheless, this study was limited by the small number of subjects and requires validation in a larger population (Table II) [102].

Interestingly, Wong et al. [103] found that expression of four miRNAs (miRNA-125a-5p, -190a, $-550 a-5 p$ and -638) could differentiate HFrEF from HFpEF. Furthermore, miRNA-183-3p, -190a, -193$3 p,-193-5 p$, and $-545-5 p$ distinguished HFpEF from non-HF controls. These authors also found that miRNA-183-3p, -190a, -193b-3p, -193b-5p, $-211-5 p,-494,-671-5 p$, and -1233 could differentiate HF from non-HF. In addition, the AUC values of individual miRNAs had a lower discriminative power in HFpEF vs. HFrEF than NT-proBNP, but the miRNA panel in combination with NT-proBNP achieved maximal diagnostic accuracy (AUC 1.0, Table II). Thus, combining assessment of individual miRNA with NT-proBNP or employing a unique panel of multiple miRNA biomarkers can signifi- cantly improve the specificity and sensitivity of diagnostic assays and accelerate HF diagnosis.

In order to identify a novel miRNA signature helping to differentiate HFpEF from HFrEF, Watson et al. [104] performed miRNA profiling from the plasma RNA pool of $15 \mathrm{HFpEF}$ patients, $15 \mathrm{HFrEF}$ patients and 15 healthy controls. Five miRNA candidates - miR-30c, miR-146a, miR-221, miR328 , and miR-375 - showing potential as HF biomarkers that could differentiate HFpEF and HFrEF were selected and further verified and validated in an independent cohort of 225 patients $(n=$ 75 per group). In this study, all five miRNAs were reduced in HF; however, miRNA-375 was only reduced in HFrEF. miRNA-328 and miRNA-375 levels were significantly different between HFpEF and HFrEF when comparing average circulating levels (Table II) [104]. Circulating miRNA-328 plays an important role in cardiac hypertrophy through mechanisms involving SERCA2a [105] and could be a potential indicator of acute myocardial infarction, and increased plasma levels of miRNA are strongly associated with increased risk of mortality and development of HF [106]. Meanwhile, miR375, which was the only down-regulated miRNA in HFpEF patients, has been linked to diabetes [107]. Similarly to the earlier study, the authors performed AUC analyses to distinguish HFpEF from HFrEF and reported equally predictive values of each of the single miRNAs compared with the use of two or more of miRNA-146a, -221, -328 and BNP. Importantly, the combination of miRNA-375 with BNP significantly improved the predictive power to differentiate HFpEF from HFrEF as compared with BNP alone in the AUC model. Again, the highest intergroup distinction was achieved by combining the miRNA panel with BNP (AUC = 0.854).

Although the recent results in miRNA-based diagnostics of HFpEF and HFrEF are highly promising, there are some limitations to clinical implementation of miRNA signature detection. The first is the lack of a strong biological correlation between circulatory miRNA levels and the relevant organ/tissue expression in HF. The next limitation concerns the fact that circulating NT-proBNP level was far superior to signature miRNAs in predicting HF. The two recent studies (see above) suggested that combining miRNA levels with NT-proBNP might add diagnostic value to differentiate HFpEF from HFrEF [108].

There is a growing body of evidence that miRNAs play a major role in the transcriptional and translational changes in gene expression with respect to cardiac hypertrophy and fibrosis in humans and mouse models of $\operatorname{HF}[109,110]$. Dysregulation of specific miRNAs could alter the cellular responses of cardiomyocytes and non-cardiomyocytes to specific signalling upon patholog- 


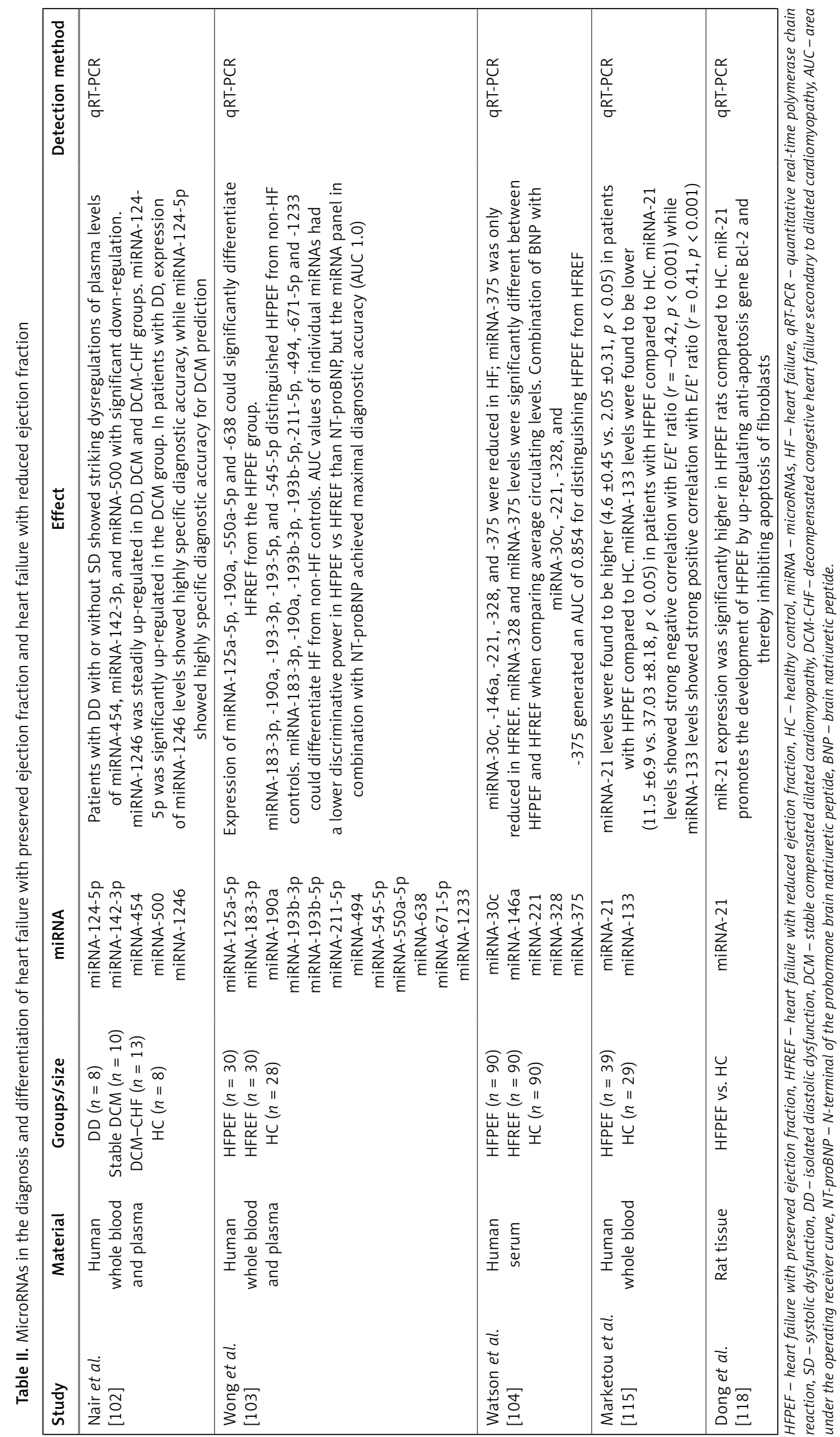


ical haemodynamic overload, leading to cardiac hypertrophy and HF [111]. Recent studies have revealed that miRNA-21 and miRNA-133 play a role in heart hypertrophy and fibrosis. They have also been shown to regulate proliferation and phenotypic switch of vascular smooth muscle cells. More recent studies validate these findings of miR-21 promoting cardiac remodelling and fibrosis [112, 113]. Also, it was demonstrated that miRNA-133, which controls cardiomyocyte identity, can restore heart function in rodents and might play an important role in heart regeneration and repair [114]. However, there are limited data regarding its role in HFpEF. Marketou et al. [115] analysed miRNA of the peripheral blood mononuclear cells in 39 patients with signs and symptoms of HF who had an LVEF > 50\% and evidence of LV diastolic dysfunction and 29 healthy volunteers for comparison. Quantitative real-time reverse transcription PCR revealed that miRNA-21 levels were higher in peripheral blood mononuclear cells (PBMCs) of HFpEF patients (4.6 \pm 0.45 vs. $2.05 \pm 0.31, p<0.05$ ), while miRNA-133 levels were lower in PBMCs of those patients $(11.5 \pm 6.9$ vs. $37.03 \pm 8.18, p<0.05)$ compared to healthy controls (Table II) [115].

In addition to developing a panel of miRNAs to function as biomarkers for diagnostic and prognostic purposes, miRNAs may be used in developing therapeutics. MicroRNA-208a is essential for cardiac hypertrophy and fibrosis. In the study of Wang et al., miRNA-208a increased endoglin expression to induce myocardial fibrosis in volume-overloaded HF [116]. A study by Montgomery et al. [117] using an animal model showed that delivery of an anti-miRNA may inhibit miRNA-208a. Subcutaneous administration of anti-miRNA208a during hypertension-induced HF in Dahl hypertensive rats prevented cardiac remodelling and improved function and survival [117]. The silencing of miRNA-208a by anti-miRNA-208a was noted to induce significant changes in cardiac gene expression, as might be expected, given that miRNAs play a large role in gene expression. In another study, Dong et al. [118] observed that after injection of an miR-21 antagonist, cardiac atrophy and cardiac fibrosis were conspicuously ameliorated in HFpEF rats. The authors suggested that miRNA-21 promoted the development of HFpEF by up-regulating the expression of the anti-apoptotic gene $\mathrm{BCl}-2$, thereby suppressing the apoptosis of cardiac fibrosis (Table II). Both in vivo and in vitro, inhibition of miRNA-21 expression resulted in reduced $\mathrm{BCl}-2$ expression while over-expression of miRNA-21 led to elevation of $\mathrm{Bcl}-2$ expression.

The use of antisense oligonucleotides to silence miRNAs could pave the way to establishing a novel avenue of target-driven therapeutics. In recent years, several opportunities to alter levels of circulating miRNAs by systemically administering agents such as mirmimics or antagomirs have been identified. This therapeutic approach has been reported to significantly reduce hypertrophy and cardiac fibrosis and to improve LV function in animal models. It represents a promising approach to complement existing therapeutic options in the treatment of HF. However, the results of in-vitro and in-vivo experiments have not yet led to application in clinical studies. Successful implementation of these insights in clinical trials represents the next step to bring this idea to fruition [119].

\section{Abnormal levels of laboratory markers and co-morbidities as a potential prognostic tool and therapeutic target in HFpEF}

Besides interventions at the level of the heart itself targeting fibrosis and myocardial stiffness, therapeutic considerations in the management of HFpEF largely rely on the management of its co-morbidities. Therefore, the identification of co-morbidities of HFpEF using diagnostic biomarkers is a very promising approach. However, cut-off values may differ from those in healthy subjects or patients with different illnesses. The list of co-morbidities in this regard is very long, but a few examples are discussed below.

Anaemia, for example, is a common comorbidity of HF, and has been associated with poor functional status, longer hospitalization duration, early re-hospitalization, and death. The incremental risks of death and lengthened hospital stay associated with anaemia are more pronounced in acute decompensated HF patients with HFpEF than those with HFrEF [120].

Martens et al. [121] evaluated 1197 patients (71\% male) including $897 \mathrm{HFrEF,} 229 \mathrm{HFmrEF}$ and $72 \mathrm{HFpEF}$ patients. The overall prevalence of ID was $53 \%$ (50\% in HFrEF; $61 \%$ in HFmrEF; $64 \%$ in HFpEF) and $36 \%$ for anaemia. ID was associated with a lower $\mathrm{VO}_{2}$ max in patients with all types of heart failure $(p<0.001$ in all). Iron status was more closely related to a poor $\mathrm{VO}_{2}$ max than anaemia status $(p<0.001)$. Furthermore, poor clinical outcome was more strongly associated with iron status than anaemia status. Patients with progression of ID exhibited a significantly higher risk of heart failure hospitalisation and all-cause mortality $(\mathrm{HR}=1.4 ; 95 \% \mathrm{Cl}: 1.01-1.94 ; p=0.046)$ than patients without progression [121].

Vullaganti et al. [122] studied 56 patients who had anaemia defined as haemoglobin $\leq 12 \mathrm{~g} / \mathrm{dl}$ (mean: $10.4 \pm 1.0 \mathrm{~g} / \mathrm{dl}$ ) with HFpEF defined as having an NHANES-CHF (National Health And Nutrition Examination Survey: Congestive Heart Failure) criteria score of $\geq 3$ and an LVEF > 40\% (mean: $63 \pm 15 \%$ ). Patients were randomly allocated to receive either epoetin alfa with ferrous 
gluconate or ferrous gluconate only. In this analysis, a responder was defined as a patient with an increase of $1 \mathrm{~g} / \mathrm{dl}$ in the first 4 weeks of the trial. Unfortunately, a significant haemoglobin response to anaemia treatment with epoetin alfa and oral iron did not lead to differences in LV remodelling, functional status, or quality of life. Given the results of this small trial, it appears as though using objective improvements in anaemia as a marker in older adult subjects with HFpEF does not have significant clinical utility [122]. Anaemia can be associated with iron deficiency (ID), but the two do not have to be present at the same time. Patients with HF and iron deficiency have shown symptomatic improvements from intravenous iron administration, and some evidence suggests that these improvements occur irrespective of the presence of anaemia [123]. Functional ID is an independent predictor of poor outcome in advanced HFrEF, but its role in HFpEF remains less clear [124]. A study by Kasner et al. [124] included 26 patients with HFpEF who showed an increase in LV stiffness as assessed using pressure-volume loop analysis obtained by conductance catheterization. HFpEF patients with serum ferritin < 100 $\mu \mathrm{g} / \mathrm{l}$ or ferritin of $100-299 \mu \mathrm{g} / \mathrm{l}$ in combination with transferrin saturation $<20 \%$ were defined as having ID [124]. According to the linear regression analysis, LV stiffness was correlated with peak oxygen uptake $(r=-0.636, p<0.001)$ but not with ferritin level or transferrin saturation. No relation was found between ID and exercise capacity. The association of LV stiffness with exercise performance was independent from the level of ID. The authors concluded that in non-anaemic HFpEF patients, cardiac dysfunction and impaired exercise capacity occur independently of ID [124]. There is still a lack of large observational studies assessing the significance of anaemia and ID in HFpEF. However, a prospective trial of intravenous ferric carboxymaltose vs. placebo is underway in patients with HFpEF in order to improve exercise capacity in these patients.

Hyponatraemia has been shown to be a relevant prognostic factor in HFpEF. Serum sodium (sNa) cut-off, however, has not been defined in HFpEF, and it remains unclear whether alternative cut-off values should be used [125]. Kusaka et al. [125] assessed cardiac function using echocardiography and measured sNa in HFpEF patients in NYHA class II $(n=321)$ or III $(n=84)$ in a compensated condition after implementing medical therapy for HF. During a mean follow-up of 27 months, 73 patients developed HF-related events. In multivariate Cox hazard analysis including established predictors in HF, sNa level as a continuous variable was identified as an independent predictor for HF-related events in HFpEF (per $1.0 \mathrm{mmol} / \mathrm{l}: \mathrm{HR}=0.93$; 95\% Cl: 0.87-0.98; $p<0.01$ ) [125]. Kaplan-Meier analysis demonstrated significantly higher probability of HF-related events in the lower sNa group ( $\mathrm{sNa}<140 \mathrm{mmol} / \mathrm{l})$ than in the higher sNa group (sNa $\geq 140 \mathrm{mmol} / \mathrm{l}$; $p<0.001)$. Further, the low-normal sNa group $(135 \mathrm{mmol} / \mathrm{l}<\mathrm{sNa}<140 \mathrm{mmol} / \mathrm{l})$ was significantly associated with HF-related events compared with the higher sNa group $(p<0.001)$ [125]. In The Korea Acute Heart Failure (KorAHF) registry of 5625 patients, hyponatraemia was defined as a serum sodium level < $135 \mathrm{mmol} / \mathrm{l}$ at hospital admission [126]. HFpEF and HFrEF in this study were defined as LVEF $\geq 50 \%$ and LVEF $\leq 40 \%$, respectively. Hyponatraemic patients had higher in-hospital mortality or urgent heart transplantation in all $(11.3 \%$ vs. $4.5 \%, p<0.001)$, in HFrEF $(13.1 \%$ vs. $4.9 \%$, $p<0.001)$, and in HFpEF (6.0\% vs. $1.9 \%, p<0.001)$ [126]. However, the long-term prognostic value of hyponatraemia was limited to patients with HFrEF, and did not apply to those with HFpEF. Both serum levels of sodium - low but still normal (135$140 \mathrm{mmol} / \mathrm{l})$ and lower than normal (<135 mmol/l) - could provide important prognostic information in patients with HFpEF.

The pathophysiological mechanisms underlying the associations between co-morbidities such as obesity, diabetes mellitus, lung diseases, chronic kidney disease, liver diseases and HFpEF remain unclear. They are partially associated with systemic inflammation, and Paulus et al. [127] hypothesized that chronic inflammation leads to endothelial dysfunction, myocardial hypertrophy, fibrosis, and diastolic dysfunction [127]. Other proposed pathophysiological mechanisms include abnormal haemodynamics, metabolic dysregulation, and neurohumoral activation [127]. Indeed, more than $80 \%$ of patients with HFpEF are overweight or obese, and in the TOPCAT and RELAX trials, median/mean BMI was $31 \mathrm{~kg} / \mathrm{m}^{2}$ and over $35 \mathrm{~kg} / \mathrm{m}^{2}$, respectively $[15,128]$. Obokata et al. [129] described a distinct obese HFpEF phenotype, characterized by greater concentric LV remodelling, higher LV filling pressures at rest and with exercise, greater plasma volume overload (yet lower NT-proBNP levels), a larger increase in pulmonary arterial pressures with exercise, larger right ventricular size, and more significant exercise intolerance compared with non-obese HFpEF [129]. In addition to inflammation, the obese insulin-resistant subjects demonstrated an increase in extracellular matrix markers such as collagen VI and matrix metalloproteinase-7 when compared with obese insulin-sensitive subjects [130]. In a large cohort of obese patients without previous HF, Sundström et al. [131] recently demonstrated a graded association between increasing weight loss and decreasing risk of incident HF, suggesting that comorbid intervention may be beneficial before HFpEF ensues [131]. Confirming this notion, 
weight loss following bariatric surgery was associated with reduced LV mass and mass-volume ratio, and improved diastolic function [132].

It is difficult to discuss co-morbidities of HF without mentioning diabetes. Based on an analysis that included 3,385 patients (mean age: 69 \pm 9.6 years; $49 \%$ male; $89 \%$ white) with HFpEF from the Treatment of Preserved Cardiac Function Heart Failure With an Aldosterone Antagonist Trial (TOPCAT), Sandesara et al. [133] concluded that diabetes is associated with adverse cardiovascular outcomes in HFpEF, and the inherent risk of adverse outcomes in HFpEF patients with diabetes varies according to the presence of microvascular complications [133]. The appropriate treatment of diabetes is also important in the context of HFpEF prognosis. The activity of the sodium-hydrogen exchanger is markedly increased in patients with $\mathrm{HF}$ and may be responsible for resistance to both diuretics and endogenous natriuretic peptides. In addition, in the heart, empagliflozin appears to inhibit sodium-hydrogen exchange, which may in turn lead to a reduction in cardiac injury, hypertrophy, fibrosis, remodelling, and systolic dysfunction. The benefits of such sodium-glucose co-transporter 2 (SGLT2) inhibitors in HF may be mediated by the inhibition of sodium-hydrogen exchange rather than the effect on glucose reabsorption. Empagliflozin was associated with a $35 \%$ reduction in the risk of HF hospitalization and a $39 \%$ reduction in the risk of HF death or hospitalization compared with placebo in patients with type 2 diabetes mellitus and cardiovascular disease in the EMPA-REG OUTCOME (Empagliflozin Cardiovascular Outcome Event Trial in Type 2 Diabetes Mellitus Patients) trial, with benefits seen in patients with and without HF at baseline [134]. HF outcomes relative to LVEF were not assessed, although HF outcome rates in those with baseline HF were similar to those in HFpEF trials, leading to suggestions that empagliflozin may have been beneficial in patients with HFpEF. The mechanisms by which empagliflozin may positively affect HF are unclear, but the improvement in HF outcomes was independent of glycaemic control. Hypotheses include a blood pressure-lowering effect, sodium and fluid loss, beneficial effects on the renin angiotensin system, weight loss, maintenance of renal function, decreased atrial stiffness, and decreased inflammation [135].

Approximately $30-40 \%$ of patients with HFpEF have chronic obstructive pulmonary disease [136]. In a large, community-based sample, Lam et al. [136] found that airflow obstruction was the most prominent non-cardiac predictor of incident HFpEF, and Barr et al. [137] showed that emphysema and airflow limitation are linearly related to impaired LV filling. Indeed, HFpEF has several pathophysiological aspects, including stiffness and/or congestion of multiple organs. Yoshihisa et al. [138] investigated the impact of non-alcoholic fatty liver disease fibrosis score (NFS; based on aspartate aminotransferase to alanine aminotransferase ratio, platelet count, and albumin) on prognosis in a prospective study based on 492 HFpEF patients, with consideration of the peripheral collagen markers. The NFS, a novel indicator of liver fibrosis, correlated with circulating systemic markers of fibrosis and congestion such as procollagen type III peptide, type IV collagen 7S, and hyaluronic acid and BNP and was independently associated with higher all-cause mortality in HFpEF patients. The NFS can be calculated in a simple manner and may be a useful tool to assess liver stiffness and prognosis in HFpEF patients [138].

Approximately $25-50 \%$ of patients with HFpEF have chronic kidney disease with an estimated glomerular filtration rate (GFR) $<60 \mathrm{ml} / \mathrm{min} / 1.73$ $\mathrm{m}^{2}$, the prevalence of which increases with age [139]. Renal impairment causes metabolic and systemic derangements in circulating factors, causing an activated systemic inflammatory state and endothelial dysfunction, which may lead to cardiomyocyte stiffening, hypertrophy, and interstitial fibrosis via cross-talk between the endothelium and cardiomyocyte compartments [140]. Patients with CKD and hypertension are prone to develop HFpEF, and myocardial fibrosis has been suggested as a major determinant of disturbances in diastolic function in these patients. In rat models, CKD was significantly correlated with interstitial myocardial fibrosis, suggesting a mechanism of action for abnormal cardiac mechanics in these patients [141]. Chronic kidney disease-associated mortality is worse in patients with HFpEF compared with patients with HFrEF even after controlling for covariates [142]. Go et al. demonstrated a stepwise increase in mortality with decreasing eGFR in HFpEF [143]. The correct assessment of GFR remains challenging. Cystatin C, for example, is a marker of renal function that also predicts cardiovascular outcomes [144]. In patients with HFpEF of hypertensive origin, cystatin $C$ has been shown to be increased and associated with diastolic dysfunction and alterations in collagen metabolism independently of eGFR. An excess of cystatin $C$ might contribute to diastolic dysfunction in HFpEF by facilitating myocardial fibrosis [144]. In a study of Li et al. [145] there was a positive correlation between serum cystatin $C$ levels and interventricular septal thickness, posterior wall thickness, and LV weight index, and the serum level of cystatin C was an independent marker for hypertensive LV hypertrophy. Moran et al. [146] examined 4453 subjects aged 65 years or older without HF at baseline from the Cardiovascular Health Study. They compared the asso- 
ciation of cystatin $\mathrm{C}$ with risk of incident HFpEF and HFrEF. During 8 years of follow-up, increased risk of HFpEF was apparent only in the highest cystatin C quartile ( $\mathrm{HR}=2.25 ; 95 \% \mathrm{Cl}: 1.33-3.80)$, while a linear trend was present for HFrEF [146]. In the study of Bielecka-Dabrowa et al., worse kidney function assessed with both eGFR and cystatin C was independently associated with worse course of $\mathrm{HF}$ with preserved and moderately reduced EF [147]. In a prospective analysis of a cohort of 299 patients with HFpEF, Unger et al. [148] found that chronic kidney disease and reduced GFR were both associated with worse cardiac mechanical indices including left atrial reservoir strain, LV longitudinal strain, and right ventricular free wall strain, even after adjusting for potential confounders, including co-morbidities, LVEF, and volume status. Reduced GFR was also associated with worse outcomes (adjusted HR = 1.28, 95\% Cl: 1.01-1.61 per 1 - SD decrease in GFR; $p=0.039$ ) [148]. Gori et al. studied 217 participants with HFpEF from the PARAMOUNT trial who had echocardiography and measures of kidney function assessed [149]. Renal dysfunction was associated with abnormal LV geometry (defined as concentric hypertrophy, or eccentric hypertrophy, or concentric remodelling), lower mid-wall fractional shortening (MWFS), and higher NT-proBNP values [149]. In these studies in HFpEF patients, chronic kidney disease was independently associated with worse cardiac mechanics, which may explain why HFpEF patients with kidney disease have worse outcomes [148, 149]. Thus, the therapeutic strategies aimed at reducing cardiac fibrosis may provide a particular cardioprotective benefit in patients with chronic kidney disease. There is evidence that long-term treatment with different loop diuretics may have a variable impact on myocardial fibrosis in chronic HF patients [150]. In fact, although torasemide treated patients exhibited a reduction in myocar- dial collagen accumulation and a diminution of collagen type I synthesis, furosemide-treated patients did not [150]. Non-cardiac factors influencing prognosis in HFpEF are presented in Figure 2.

\section{Conclusions}

The diagnosis of HFpEF remains challenging, especially when the patient presents in an outpatient setting without obvious signs of volume overload. In this context, biomarkers, with their objectivity, reproducibility and accessibility, would be excellent additions to imaging studies in HFpEF diagnosis and useful to tailor therapies to an individual's unique risk profile [39, 151-155]. Currently, the only guideline-recommended biomarkers in this regard are NT-BNP and NT-proBNP. Indeed, studies suggest that natriuretic peptides have the potential to improve even the diagnosis of early HFpEF, but they still have significant limitations. There remain doubts whether the cut-off points of NT-proBNP and BNP in diagnosis and monitoring should be the same across the entire HF spectrum. Based on evidence from endomyocardial biopsies, some of the specific cardiac structural phenotypes to be targeted in HFPEF may be represented by myocyte hypertrophy, interstitial fibrosis, and myocardial inflammation. Hence biomarkers reflecting these mechanisms may be useful in HFpEF.

The new therapies should be targeted towards specific HF phenotypes, instead of the 'one-sizefits-all' approach, which has not been successful in clinical HFpEF. Unless the structural and biological determinants of the failing heart are deeply understood, it will be impossible to differentiate HFpEF patients appropriately, identify subtle myocardial abnormalities, and finally reverse abnormal cardiac function. The identification of specific structural cardiac HFpEF phenotypes may represent one way to differentiate subgroups of HFPEF patients with specific therapeutic targets. The use of selec-

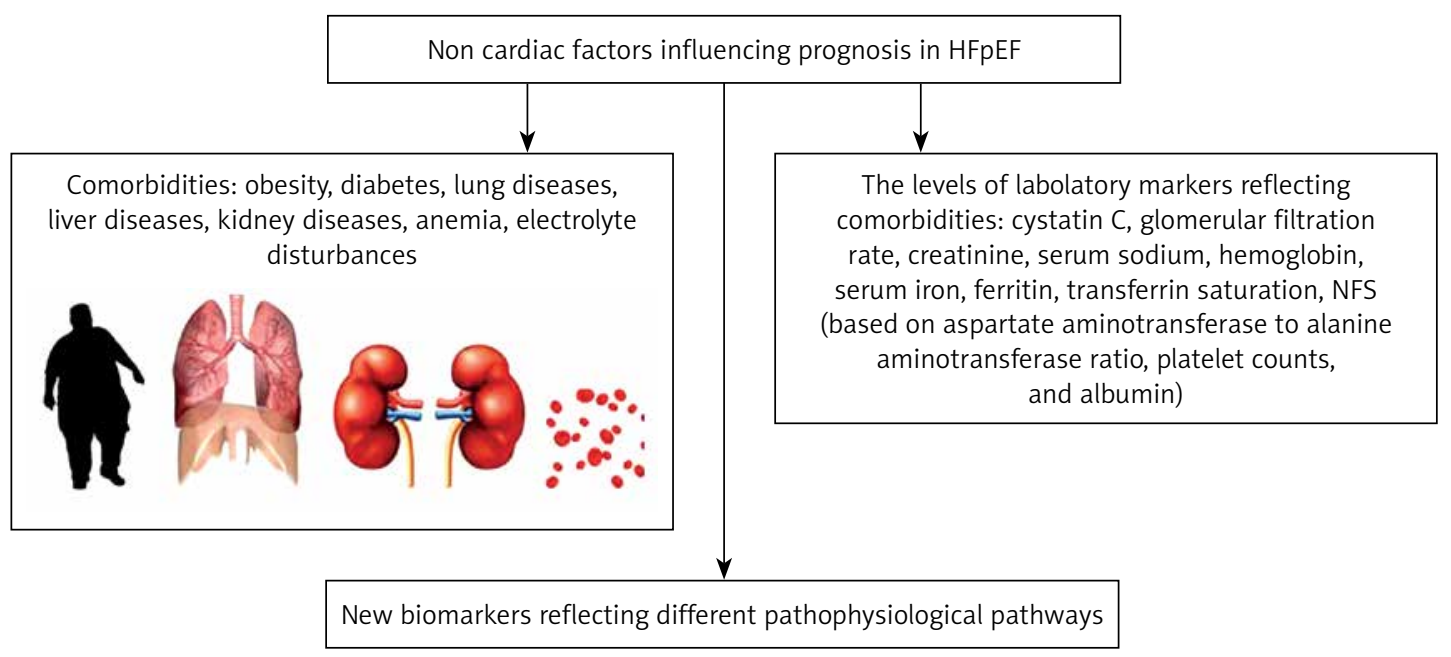

Figure 2. Non-cardiac factors influencing prognosis in HFpEF 
tive biomarkers in HFpEF may help to distinguish pathophysiological mechanisms of HFpEF [20].

Concentrating on biomarkers of fibrosis and inflammation, SST2, GDF-15, galectin-3 and other new cardiac markers associated with diastolic dysfunction and disturbed left ventricular filling have emerged, but still evidence from large cohorts of patients with HFpEF is lacking. In particular, there are no clinical studies to suggest cut-off values that can help in clinical decision making for these markers. Furthermore, the field of miRNAs is a very promising area of research, and further exploration of miRNAs may offer diagnostic and prognostic applications and insights into pathology pointing to new phenotype-specific therapeutic targets [156]. This is especially interesting considering the fact that their molecular construction and integration in cellular and intercellular transport mechanisms define miRNAs as ideal circulating biomarkers. Nevertheless, looking at the large number of studies, only a few of them have confirmed previous findings with identical results, and still different miRNAs are being identified as linked to HF, presumably reflecting the complex interactions of miRNAs and their target sites. An important aspect when performing miRNA analyses is the comparability and standardization of analytical methods and the need for well-defined study samples [157, 158]. In our opinion, biomarkers of fibrosis or inflammation cannot replace natriuretic peptides for the diagnosis of HFpEF, but they may provide additive value, especially for assessing aggravation and prognosis in these patients. In our view, results from large cohorts and meta-analyses are required to establish the role of combinations of biomarkers as clinically applicable diagnostic and/or prognostic tools in HFpEF. Characterization of the biological phenotypes, understanding the biological differences between patients with typical comorbidities but without HF and those with clinically similar profiles but with $\mathrm{HF}$, and identification of interventions that disrupt or reverse this pathobiology, may pave the way for patients to be targeted before the HFpEF syndrome ensues.

In describing the selected biomarkers of fibrosis and inflammation SST2, GDF-15, galectin-3, and miRNAs associated with diastolic dysfunction and disturbed left ventricular filling, we note that there is still a lack of evidence from large cohorts of patients with HFpEF. In this review we excluded markers of interstitial matrix turnover, because we presented them in a previous paper to which the reader is referred [39]. There have not been sufficient studies assessing the utility of biomarkers of fibrosis or inflammation in HFpEF to specify their role in only one phenotype. It is worth noting that the concentrations of biomarkers indicat- ing the diagnosis of HFpEF vary in patients with acute versus chronic compensated HF [149]. For example, threshold values of BNP and NT-proBNP validated for diagnosis of undifferentiated acutely decompensated HF remain useful in patients with HFpEF, with minor loss of diagnostic performance. In stable treated HF, plasma natriuretic peptide concentrations often fall below cut-off values used for diagnosis of acute decompensated HF in the emergency department; in HFpEF, levels average approximately half of those in HFpEF [149].

\section{Acknowledgments}

Marta Michalska-Kasiczak and Agata BieleckaDabrowa contributed equally to this work.

\section{Conflict of interest}

$M M K, A G B$, and MB have not received any payments in connection with the preparation of this review. SvH has been a paid consultant to Roche, BRAHMS/Thermo Fisher Scientific, Vifor, and Bayer. He has received speaker's fees from Boehringer Ingelheim, Novartis, and Amgen. No medical or pharmaceutical company was involved in the preparation of this article.

\section{References}

1. Lindenfeld J, Albert NM, Boehmer JP, et al. Executive Summary: HFSA 2010 Comprehensive Heart Failure Practice Guideline. J Card Fail 2010; 16: 475-53.

2. Chronic Heart Failure. National Clinical Guideline for Diagnosis and Management in Primary and Secondary Care. (National Clinical Guideline Centre, 2010). http:// www.ncbi.nlm.nih.gov/pubmedhealth/PMH0046954/.

3. Taylor R, Sagar VA, Davies EJ. et al. Exercise-based rehabilitation for heart failure. Cochrane Database Syst Rev 2014; 4: CD003331.

4. Ponikowski P, Voors AA, Anker SD, et al. 2016 ESC Guidelines for the diagnosis and treatment of acute and chronic heart failure: The Task Force for the diagnosis and treatment of acute and chronic heart failure of the European Society of Cardiology (ESC). Developed with the special contribution of the Heart Failure Association (HFA) of the ESC. Eur J Heart Fail 2016; 18: 891-975.

5. Paulus WJ, Tschope C, Sanderson JE, et al. How to diagnose diastolic heart failure: a consensus statement on the diagnosis of heart failure with normal left ventricular ejection fraction by the Heart Failure and Echocardiography Associations of the European Society of Cardiology. Eur Heart J 2007; 28: 2539-50.

6. Vasan RS, Levy D. Defining diastolic heart failure: a call for standardized diagnostic criteria. Circulation 2000; 101: 2118-21.

7. McMurray JJ, Adamopoulos S, Anker SD, et al. ESC Guidelines for the diagnosis and treatment of acute and chronic heart failure 2012: The Task Force for the Diagnosis and Treatment of Acute and Chronic Heart Failure 2012 of the European Society of Cardiology. Developed in collaboration with the Heart Failure Association (HFA) of the ESC. Eur Heart J 2012; 33: 1787-847. 
8. Oghlakian GO, Sipahi I, Fang JC. Treatment of heart failure with preserved ejection fraction: have we been pursuing the wrong paradigm? Mayo Clin Proc 2011; 86: 531-9.

9. Yusuf S, Pfeffer MA, Swedberg K, et al.; CHARM Investigators and Committees. Effects of candesartan in patients with chronic heart failure and preserved left-ventricular ejection fraction: the CHARM-Preserved Trial. Lancet 2003; 362: 777-81.

10. Massie BM, Carson PE, McMurray JJ, et al.; I-PRESERVE Investigators. Irbesartan in patients with heart failure and preserved ejection fraction. N Engl J Med 2008; 359: 2456-67.

11. Cleland JG, Tendera M, Adamus J, Freemantle N, Polonski L, Taylor J; PEP-CHF Investigators. The perindopri in elderly people with chronic heart failure (PEP-CHF) study. Eur Heart J 2006; 27: 2338-45.

12. Pitt B, Pfeffer MA, Assmann SF, et al.; TOPCAT Investigators. Spironolactone for heart failure with preserved ejection fraction. N Engl J Med 2014; 370: 1383-92.

13. Eyre V, Lang CC, Smith K, et al.; REACH-HF investigators. Rehabilitation Enablement in Chronic Heart Failure - a facilitated self-care rehabilitation intervention in patients with heart failure with preserved ejection fraction (REACH-HFpEF) and their caregivers: rationale and protocol for a single-centre pilot randomised controlled trial. BMJ Open 2016; 6: e012853.

14. Fu M, Zhou J, Thunström E, et al. Optimizing the management of heart failure with preserved ejection fraction in the elderly by targeting comorbidities (OPTIMIZEHFPEF). J Card Fail 2016; 22: 539-44.

15. Owan TE, Hodge DO, Herges RM, Jacobsen SJ, Roger VL, Redfield MM. Trends in prevalence and outcome of heart failure with preserved ejection fraction. N Engl J Med 2006; 355: 251-9.

16. Metra M. June 2016 at a glance: epidemiology, renal impairment, heart failure with preserved ejection fraction. Eur J Heart Fail 2016; 18: 587.

17. Primessnig U, Schönleitner P, Höll A, et al. Novel pathomechanisms of cardiomyocyte dysfunction in a model of heart failure with preserved ejection fraction. Eur J Heart Fail 2016; 18: 987-97.

18. Hogg K, Swedberg K, McMurray J. Heart failure with preserved left ventricular systolic function: epidemiology, clinical characteristics, and prognosis. J Am Coll Cardiol 2004; 43: 317-27.

19. Oktay AA, Rich JD, Shah SJ. The emerging epidemic of heart failure with preserved ejection fraction. Curr Heart Fail Rep 2013; 10: 401-10.

20. D’Elia E, Vaduganathan M, Gori M, Gavazzi A, Butler J, Senni M. Role of biomarkers in cardiac structure phenotyping in heart failure with preserved ejection fraction: critical appraisal and practical use. Eur J Heart Fail 2015; 17: 1231-9.

21. Zile MR, Baicu CF, Gaasch WH. Diastolic heart failureabnormalities in active relaxation and passive stiffness of the left ventricle. N Engl J Med 2004; 350: 1953-9.

22. van Heerebeek L, Paulus WJ. Impact of comorbidities on myocardial remodeling and dysfunction in heart failure with preserved ejection fraction. SOJ Pharm Pharm Sci 2014; 1: 1-20.

23. Linke WA, Hamdani N. Gigantic business: titin properties and function through thick and thin. Circ Res 2014; 114: 1052-68.

24. Zile MR, Baicu CF, Ikonomidis JS, et al. Myocardial stiffness in patients with heart failure and a preserved ejection fraction contributions of collagen and titin. Circulation 2015; 131: 1247-5.

25. Hidalgo C, Hudson B, Bogomolovas J, et al. PKC phosphorylation of titin's PEVK element. A novel and conserved pathway for modulating myocardial stiffness. Circ Res 2009; 105: 631-8.

26. Borbely A, Falcao-Pires I, van Heerebeek L, et al. Hypophosphorylation of the stiff N2B titin isoform raises cardiomyocyte resting tension in failing human myocardium. Circ Res 2009; 104: 780-6.

27. Bielecka-Dabrowa A, Michalska-Kasiczak M, Gluba A, et al. Biomarkers and echocardiographic predictors of myocardial dysfunction in patients with hypertension. Sci Rep 2015; 5: 8916.

28. Yancy CW, Lopatin M, Stevenson LW, De Marco T, Fonarow GC, ADHERE Scientific Advisory Committee and Investigators. Clinical presentation, management, and in-hospital outcomes of patients admitted with acute decompensated heart failure with preserved systolic function: a report from the Acute Decompensated Heart Failure National Registry (ADHERE) Database. J Am Coll Cardiol 2006; 47: 76-84.

29. Fonorow GC, Stough WG, Abraham WT, et al. OPTIMIZE-HF Investigators and Hospitals. Characteristics, treatments and outcomes of patients with preserved systolic function hospitalized for heart failure: a report from the OPTIMIZE-HF Registry. J Am Coll Cardiol 2007; 50: 768-77.

30. Lam CS, Donal E, Kraigher-Krainer E, Vasan RS. Epidemiology and clinical course of heart failure with preserved ejection fraction. Eur J Heart Fail 2011; 13: 18-28.

31. Paulus WJ, Tschöpe C. A novel paradigm for heart failure with preserved ejection fraction: comorbidities drive myocardial dysfunction and remodeling through coronary microvascular endothelial inflammation. J Am Coll Cardiol 2013; 62: 263-71.

32. Lewis GA, Schelbert EB, Williams SG, et al. Biological phenotypes of heart failure with preserved ejection fraction. J Am Coll Cardiol 2017; 70: 2186-200.

33. Schelbert EB, Fridman Y, Wong TC, et al. Temporal relation between myocardial fibrosis and heart failure with preserved ejection fraction: association with baseline disease severity and subsequent outcome. JAMA Cardiol 2017; 2: 995-1006.

34. Berezin AE. Prognostication in different heart failure phenotypes: the role of circulating biomarkers. J Circ Biomark 2016; 5: 6.

35. Zile MR, Baicu CF. Biomarkers of diastolic dysfunction and myocardial fibrosis: application to heart failure with a preserved ejection fraction. J Cardiovasc Transl Res 2013; 6: 501-15.

36. Linde C, Eriksson MJ, Hage C, et al.; Stockholm County/Karolinska Institutet 4D heart failure investigators. Rationale and design of the PREFERS (Preserved and Reduced Ejection Fraction Epidemiological Regional Study) Stockholm heart failure study: an epidemiological regional study in Stockholm county of 2.1 million inhabitants. Eur J Heart Fail 2016; 18: 1287-97.

37. Toma M, Mak GJ, Chen V, et al. Differentiating heart failure phenotypes using sex-specific transcriptomic and proteomic biomarker panels. ESC Heart Fail 2017; 4: 301-11.

38. Sharma K, Kass DA. Heart failure with preserved ejection fraction: mechanisms, clinical features, and therapies. Circ Res 2014; 115: 79-96.

39. Gluba A, Bielecka-Dabrowa A, Mikhailidis DP, et al. An update on biomarkers of heart failure in hypertensive patients. J Hypertens 2012; 30: 1681-9. 
40. Palmer SC, Yandle TG, Nicholls MG, Frampton CM, Richards AM. Regional clearance of amino-terminal pro-brain natriuretic peptide from human plasma. Eur J Heart Fail 2009; 11: 832-9.

41. O'Meara E, de Denus S, Rouleau JL, Desai A. Circulating biomarkers in patients with heart failure and preserved ejection fraction. Curr Heart Fail Rep 2013; 10: 350-8.

42. Balion CM, Santaguida P, McKelvie R, et al. Physiological, pathological, pharmacological, biochemical and hematological factors affecting BNP and NT-proBNP. Clin Biochem 2008; 41: 231-9.

43. Bishu K, Deswal A, Chen HH, et al. Biomarkers in acutely decompensated heart failure with preserved or reduced ejection fraction. Am Heart J 2012; 164: 763-70.

44. Sanders-van Wijk S, van Empel V, Davarzani N, et al.; TIME-CHF investigators. Circulating biomarkers of distinct pathophysiological pathways in heart failure with preserved vs. reduced left ventricular ejection fraction. Eur J Heart Fail 2015; 17: 1006-14.

45. van Veldhuisen DJ, Linssen GC, Jaarsma T, et al. B-type natriuretic peptide and prognosis in heart failure patients with preserved and reduced ejection fraction. J Am Coll Cardiol 2013; 61: 1498-506.

46. Li P, Wang D, Lucas J, et al. Atrial natriuretic peptide inhibits transforming growth factor beta-induced Smad signaling and myofibroblast transformation in mouse cardiac fibroblasts. Circ Res 2008; 102: 185-92.

47. Zile MR, Little WC. Heart failure with a preserved ejection fraction. In: Braunwald's Heart Disease: A Textbook of Cardiovascular Medicine. Mann D, Zipes D, Libby P, Bonow R (eds). 10 ${ }^{\text {th }}$ ed. Elsevier, 2014; 564.

48. Carlsen CM, Bay M, Kirk V, Gotze JP, Kober L, Nielsen OW. Prevalence and prognosis of heart failure with preserved ejection fraction and elevated N-terminal probrain natriuretic peptide: a 10-year analysis from the Copenhagen Hospital Heart Failure Study. Eur J Heart Fail 2012; 14: 240-7.

49. Jhund PS, Anand IS, Komajda M, et al. Changes in $\mathrm{N}$-terminal pro-B-type natriuretic peptide levels and outcomes in heart failure with preserved ejection fraction: an analysis of the I-Preserve study. Eur J Heart Fail 2015; 17: 809-17.

50. Iwanaga Y, Nishi I, Furuichi S, et al. B-type natriuretic peptide strongly reflects diastolic wall stress in patients with chronic heart failure: comparison between systolic and diastolic heart failure. J Am Coll Cardiol 2006; 47: 742-8.

51. Mukoyama M, Nakao K, Hosoda K, et al. Brain natriuretic peptide as a novel cardiac hormone in humans. Evidence for an exquisite dual natriuretic peptide system, atrial natriuretic peptide and brain natriuretic peptide. J Clin Invest 1991; 87: 1402-12.

52. Rosenzweig A, Seidman CE. Atrial natriuretic factor and related peptide hormones. Annu Rev Biochem 1991; 60: 229-55.

53. Loncar G, Omersa D, Cvetinovic N, Arandjelovic A, Lainscak $M$. Emerging biomarkers in heart failure and cardiac cachexia. Int J Mol Sci 2014; 15: 23878-96.

54. Andersen M, Ersboll M, Bro-Jeppesen J, et al. Relationships between biomarkers and left ventricular filling pressures at rest and during exercise in patients after myocardial infarction. J Card Fail 2014; 20: 959-67.

55. Shah KB, Kop WJ, Christenson RH, et al. Prognostic utility of ST2 in patients with acute dyspnea and preserved left ventricular ejection fraction. Clin Chem 2011; 57: 874-82.
56. Schmitz J, Owyang A, Oldham E, et al. IL-33, an interleukin-1-like cytokine that signals via the IL-1 receptor related protein ST2 and induces T helper type 2-associated cytokines. Immunity 2005; 23: 479-90.

57. Seki K, Sanada S, Kudinova AY, et al. Interleukin-33 prevents apoptosis and improves survival after experimental myocardial infarction through ST2 signaling. Circ Heart Fail 2009; 2: 684-91.

58. Sabatine MS, Morrow DA, Higgins LJ, MacGillivray C, Guo W, Bode C. Complementary roles for biomarkers of biomechanical strain ST2 and N-terminal prohormone B-type natriuretic peptide in patients with ST-elevation myocardial infarction. Circulation 2008; 117 1936-44.

59. Pascual-Figal DA, Ordonez-Llanos J, Tornel PL, et al. Soluble ST2 for predicting sudden cardiac death in patients with chronic heart failure and left ventricular systolic dysfunction. J Am Coll Cardiol 2009; 54: 2174-9.

60. Santhanakrishnan R, Chong JP, Ng TP, et al. Growth differentiation factor 15, ST2, high-sensitivity troponin T, and $\mathrm{N}$-terminal pro brain natriuretic peptide in heart failure with preserved vs. reduced ejection fraction. Eur J Heart Fail 2012; 14: 1338-47

61. Wang YC, Yu CC, Chiu FC, et al. Soluble ST2 as a biomarker for detecting stable heart failure with a normal ejection fraction in hypertensive patients. J Card Fail 2013; 19: 163-8.

62. Jhund PS, Claggett BL, Zile MR, et al. Soluble ST2 is associated with markers of diastolic dysfunction in patients with heart failure with preserved ejection fraction in the PARAMOUNT trial. Eur Heart J 2014; 35: 340-1 (Abstract).

63. Friões F, Lourenco P, Laszczynska O, et al. Prognostic value of SST2 added to BNP in acute heart failure with preserved or reduced ejection fraction. Clin Res Cardiol 2015; 104: 491-9.

64. Manzano-Fernandez S, Mueller T, Pascual-Figal D, Truong QA, Januzzi JL. Usefulness of soluble concentrations of interleukin family member ST2 as predictor of mortality in patients with acutely decompensated heart failure relative to left ventricular ejection fraction. Am J Cardiol 2011; 107: 259-67.

65. Parikh RH, Seliger SL, Christenson R, Gottdiener JS, Psaty BM, deFilippi CR. Soluble ST2 for prediction of heart failure and cardiovascular death in an elderly, community-dwelling population. J Am Heart Assoc 2016; 5: pii: e003188

66. Zhou YM, Li MJ, Zhou YL, Ma LL, Yi X. Growth differentiation factor-15 (GDF-15), novel biomarker for assessing atrial fibrosis in patients with atrial fibrillation and rheumatic heart disease. Int J Clin Exp Med 2015; 8: 21201-7.

67. Lok SI, Winkens B, Goldschmeding R, et al. Circulating growth differentiation factor-15 correlates with myocardial fibrosis in patients with non-ischaemic dilated cardiomyopathy and decreases rapidly after left ventricular assist device support. Eur J Heart Fail 2012; 14: 1249-56.

68. Kempf T, Eden M, Strelau J, et al. The transforming growth factor-beta superfamily member growth-differentiation factor-15 protects the heart from ischemia/ reperfusion injury. Circ Res 2006; 98: 351-60.

69. Kempf T, Wollert KC. Growth-differentiation factor-15 in heart failure. Heart Fail Clin 2009; 5: 537-47.

70. Lind L, Wallentin L, Kempf T, et al. Growth-differentiation factor-15 is an independent marker of cardiovascular dysfunction and disease in the elderly: results 
from the Prospective Investigation of the Vasculature in Uppsala Seniors (PIVUS) study. Eur Heart J 2009; 30: 2346-53.

71. Xue H, Fu Z, Chen Y, et al. The association of growth differentiation factor-15 with left ventricular hypertrophy in hypertensive patients. PLoS One 2012; 7: 46534.

72. Hanatani S, Izumiya Y, Takashio S, et al. Growth differentiation factor 15 can distinguish between hypertrophic cardiomyopathy and hypertensive hearts. Heart Vessels 2014; 29: 231-7.

73. Izumiya Y, Hanatani S, Kimura Y, et al. Growth differentiation factor-15 is a useful prognostic marker in patients with heart failure with preserved ejection fraction. Can J Cardiol 2014; 30: 338-44.

74. Sinning C, Kempf T, Schwarzl M, et al. Biomarkers for characterization of heart failure - distinction of heart failure with preserved and reduced ejection fraction. Int J Cardiol 2017; 227: 272-7.

75. Chan MM, Santhanakrishnan R, Chong JP, et al. Growth differentiation factor 15 in heart failure with preserved vs. reduced ejection fraction. Eur J Heart Fail 2016; 18: 81-8.

76. Phan TT, Shivu GN, Abozguia K, Sanderson JE, Frenneaux $M$. The pathophysiology of heart failure with preserved ejection fraction: from molecular mechanism to exercise haemodynamics. Int I Cardiol 2012; 158: 337-43.

77. Kopytsya M, Vyshnevska I, Protsenko O, Barahmeh H. Growth differentiation factor 15 as a prognostic marker of chronic heart failure progression in long-term follow-up after acute coronary syndrome. Georgian Med News 2017; 271: 61-6.

78. Zile MR, Brutsaert DL. New concepts in diastolic dysfunction and diastolic heart failure: part II: causal mechanisms and treatment. Circulation 2002; 105: 1503-8.

79. Borlaug BA, Paulus WJ. Heart failure with preserved ejection fraction: pathophysiology, diagnosis, and treatment. Eur Heart J 2011; 32: 670-9.

80. de Boer RA, Edelmann F, Cohen-Solal A, Mamas MA, Maisel A, Pieske B. Galectin-3 in heart failure with preserved ejection fraction. Eur J Heart Fail 2013; 15: 1095-101.

81. de Boer RA, Voors AA, Muntendam P, van Gilst WH, van Veldhuisen DJ. Galectin-3: a novel mediator of heart failure development and progression. Eur J Heart Fail 2009; 11: 811-7.

82. Calvier L, Miana M, Reboul P, et al. Galectin-3 mediates aldosterone-induced vascular fibrosis. Arterioscler Thromb Vasc Biol 2013; 33: 67-75.

83. Yu L, Ruifrok WP, Meissner M, et al. Genetic and pharmacological inhibition of galectin-3 prevents cardiac remodeling by interfering with myocardial fibrogenesis. Circ Heart Fail 2013; 6: 107-17.

84. Wu CK, Su MY, Lee JK, et al. Galectin-3 level and the severity of cardiac diastolic dysfunction using cellular and animal models and clinical indices. Sci Rep 2015; 5: 17007.

85. van Kimmenade RR, Januzzi JL Jr, Ellinor PT, et al. Utility of amino-terminal pro-brain natriuretic peptide, galectin-3, and apelin for the evaluation of patients with acute heart failure. J Am Coll Cardiol 2006; 48: 1217-24.

86. Shah RV, Chen-Tournoux AA, Picard MH, van Kimmenade RR, Januzzi JL. Galectin-3, cardiac structure and function, and long-term mortality in patients with acutely decompensated heart failure. Eur J Heart Fail 2010; 12: 826-32.

87. de Boer RA, Lok DJ, Jaarsma T, et al. Predictive value of plasma galectin-3 levels in heart failure with reduced and preserved ejection fraction. Ann Med 2011; 43: 60-8.

88. Carrasco-Sanchez FJ, Aramburu-Bodas O, SalamancaBautista P, et al. Predictive value of serum galectin-3 levels in patients with acute heart failure with preserved ejection fraction. Int J Cardiol 2013; 169: 177-82.

89. Anand IS, Rector TS, Kuskowski M, Adourian A, Muntendam P, Cohn JN. Baseline and serial measurements of galectin-3 in patients with heart failure: relationship to prognosis and effect of treatment with valsartan in the Val-HeFT. Eur J Heart Fail 2013; 15: 511-8.

90. Gullestad L, Ueland T, Kjekshus J, et al. Galectin-3 predicts response to statin therapy in the Controlled Rosuvastatin Multinational Trial in Heart Failure (CORONA). Eur Heart J 2012; 33: 2290-6.

91. Edelmann F, Holzendorf V, Wachter R, et al. Galectin-3 in patients with heart failure with preserved ejection fraction: results from the Aldo-DHF trial. Eur J Heart Fail 2015; 17: 214-23.

92. AbouEzzeddine OF, Haines P, Stevens S, et al. Galectin-3 in heart failure with preserved ejection fraction. A RELAX trial substudy (Phosphodiesterase- 5 Inhibition to Improve Clinical Status and Exercise Capacity in Diastolic Heart Failure). JACC Heart Fail 2015; 3: 245-52.

93. Bartel DP. MicroRNAs: genomics, biogenesis, mechanism, and function. Cell 2004; 116: 281-97.

94. Thum T, Galuppo P, Wolf C, et al. MicroRNAsin the human heart: a clue to fetal gene reprogramming in heart failure. Circulation 2007; 116: 258-67.

95. Thum T, Gross C, Fiedler J, et al. MicroRNA-21 contributes to myocardial disease by stimulating MAP kinase signalling in fibroblasts. Nature 2008; 456: 980-4.

96. He L, Hannon GJ. MicroRNAs: small RNAs with a big role in gene regulation. Nat Rev Genet 2004; 5: 522-31.

97. Redis RS, Calin S, Yang Y, You MJ, Calin GA. Cell-to-cell miRNA transfer: from body homeostasis to therapy. Pharmacol Ther 2012; 136: 169-74.

98. Schmitter D, Cotter G, Voors AA. Clinical use of novel biomarkers in heart failure: towards personalized medicine. Heart Fail Rev 2014; 19: 369-81.

99. Goren Y, Kushnir M, Zafrir B, Tabak S, Lewis BS, Amir O. Serum levels of microRNAs in patients with heart failure. Eur J Heart Fail 2012; 14: 147-54.

100. Dickinson BA, Semus HM, Montgomery RL, et al. Plasma microRNAs serve as biomarkers of therapeutic efficacy and disease progression in hypertension-induced heart failure. Eur J Heart Fail 2013; 15: 650-9.

101. Kondkar AA, Abu-Amero KK. Utility of circulating microRNAs as clinical biomarkers for cardiovascular diseases. Biomed Res Int 2015; 2015: 821823.

102. Nair N, Kumar S, Gongora E, Gupta S. Circulating miRNA as novel markers for diastolic dysfunction. Mol Cell Biochem 2013; 376: 33-40.

103. Wong LL, Armugam A, Sepramaniam S, et al. Circulating microRNAs in heart failure with reduced and preserved left ventricular ejection fraction. Eur J Heart Fail 2015; 17: 393-404.

104. Watson CJ, Gupta SK, O'Connell E, et al. MicroRNA signatures differentiate preserved from reduced ejection fraction heart failure. Eur J Heart Fail 2015; 17: 405-15.

105. Li C, Li X, Gao X, et al. MicroRNA-328 as a regulator of cardiac hypertrophy. Int J Cardiol 2014; 173: 268-76. 
106. He F, Lv P, Zhao X, et al. Predictive value of circulating miR-328 and miR-134 for acute myocardial infarction. Mol Cell Biochem 2014; 394: 137-44.

107. Li X. MiR-375, a microRNA related to diabetes. Gene 2014; 533: 1-4

108. Berezin AE. Predicting heart failure phenotypes using cardiac biomarkers: hype and hope. J Dis Markers 2015; 2: 1035-41.

109. van Rooij E, Sutherland LB, Liu N, et al. A signature pattern of stress-responsive microRNAs that can evoke cardiac hypertrophy and heart failure. Proc Natl Acad Sci USA 2006; 103: 18255-60.

110. Bauersachs J. Regulation of myocardial fibrosis by microRNAs. J Cardiovasc Pharmacol 2010; 56: 454-9.

111. Dong DI, Yang BF. Role of microRNAs in cardiac hypertrophy, myocardial fibrosis and heart failure. Acta Pharm Sin B 2011; 1: 1-7.

112. Liang $\mathrm{H}$, Zhang $\mathrm{C}$, Ban $\mathrm{T}$, et al. A novel reciprocal loop between microRNA-21 and TGFbetaRIII is involved in cardiac fibrosis. Int J Biochem Cell Biol 2012; 44: 2152-60.

113. Villar AV, García R, Merino D, et al. Myocardial and circulating levels of microRNA-21 reflect left ventricular fibrosis in aortic stenosis patients. Int I Cardiol 2013; 167: 2875-81.

114. Chaturvedi P, Tyagi SC. Epigenetic mechanisms underlying cardiac degeneration and regeneration. Int J Cardiol 2014; 173: 1-11.

115. Marketou M, Kontaraki J, Parthenakis F, et al. MiR-21 and miR-133 levels in peripheral blood mononuclear cells in patients with heart failure with preserved ejection fraction. Eur Heart J 2015; 36: 1028 (Abstract).

116. Wang BW, Wu GJ, Cheng WP, Shyu KG. MicroRNA-208a increases myocardial fibrosis via endoglin in volume overloading heart. PLoS One 2014; 9: e84188.

117. Montgomery RL, Hullinger TG, Semus HM, et al. Therapeutic inhibition of miR-208a improves cardiac func tion and survival during heart failure. Circulation 2011 124: 1537-47.

118. Dong S, Ma W, Hao B, et al. microRNA-21 promotes cardiac fibrosis and development of heart failure with preserved left ventricular ejection fraction by up-regulating Bcl-2. Int J Clin Exp Pathol 2014; 7: 565-74.

119. Schulte C, Westermann D, Blankenberg S, Zeller T. Diagnostic and prognostic value of circulating microRNAs in heart failure with preserved and reduced ejection fraction. World J Cardiol 2015; 7: 843-60.

120. Caughey MC, Avery CL, Ni H, et al. Outcomes of patients with anemia and acute decompensated heart failure with preserved versus reduced ejection fraction (From the ARIC Study Community Surveillance) Am J Cardiol 2014; 114: 1850-4.

121. Martens P, Nijst P, Verbrugge FH, Smeets K, Dupont M, Mullens W. Impact of iron deficiency on exercise capacity and outcome in heart failure with reduced, midrange and preserved ejection fraction. Acta Cardiol 2018; 73: 115-23.

122. Vullaganti S, Goldsmith J, Teruya S, Alvarez J, Helmke S, Maurer MS. Cardiovascular effects of hemoglobin response in patients receiving epoetin alfa and oral iron in heart failure with a preserved ejection fraction. J Geriatr Cardiol 2014; 11: 100-5.

123. von Haehling S, Jankowska EA, van Veldhuisen DJ, Ponikowski P, Anker SD. Iron deficiency and cardiovascular disease. Nat Rev Cardiol 2015; 12: 659-69.

124. Kasner M, Aleksandrov AS, Westermann D, et al. Functional iron deficiency and diastolic function in heart failure with preserved ejection fraction. Int I Cardiol 2013; 168: 4652-7.

125. Kusaka H, Sugiyama S, Yamamoto E, et al. Low-normal serum sodium and heart failure-related events in patients with heart failure with preserved left ventricular ejection fraction. Circ J 2016; 80: 411-7.

126. Park JJ, Cho YJ, Oh IY, et al. Short and long-term prognostic value of hyponatremia in heart failure with preserved ejection fraction versus reduced ejection fraction: an analysis of the Korean Acute Heart Failure registry. Int J Cardiol 2017; 248: 239-45.

127. Paulus WJ, Dal Canto E. Distinct myocardial targets for diabetes therapy in heart failure with preserved or reduced ejection fraction. JACC Heart Fail 2018; 6: 1-7.

128. Redfield MM, Chen HH, Borlaug BA, et al. Effect of phosphodiesterase- 5 inhibition on exercise capacity and clinical status in heart failure with preserved ejection fraction. JAMA 2013; 309: 1268-77.

129. Obokata M, Reddy YNV, Pislaru SV, et al. Evidence supporting the existence of a distinct obese phenotype of heart failure with preserved ejection fraction. Circulation 2017; 136: 6-19.

130. Lawler HM, Underkofler CM, Kern PA, Erickson C, Bredbeck B, Rasouli N. Adipose tissue hypoxia, inflammation, and fibrosis in obese insulin-sensitive and obese insulin-resistant subjects. J Clin Endocrinol Metab 2016; 101: 1422-8.

131. Sundström J, Bruze G, Ottosson J, Marcus C, Näslund I, Neovius M. Weight loss and heart failure: a nationwide study of gastric bypass surgery versus intensive lifestyle treatment. Circulation 2017; 135: 1577-85.

132. Aggarwal R, Harling L, Efthimiou E, Darzi A, Athanasiou T, Ashrafian $\mathrm{H}$. The effects of bariatric surgery on cardiac structure and function: a systematic review of cardiac imaging outcomes. Obes Surg 2015; 26: 1030-40.

133. Sandesara PB, O'Neal WT, Kelli HM, et al. The prognostic significance of diabetes and microvascular complications in patients with heart failure with preserved ejection fraction. Diabetes Care 2018; 41: 150-5.

134. Verma S, Mazer CD, Al-Omran M, et al. Cardiovascular outcomes and safety of empagliflozin in patients with type 2 diabetes mellitus and peripheral artery disease: a subanalysis of EMPA-REG OUTCOME. Circulation 2018; 137: 405-7.

135. Packer M, Anker SD, Butler J, Filippatos G, Zannad F. Effects of sodium-glucose cotransporter 2 inhibitors for the treatment of patients with heart failure: proposal of a novel mechanism of action. JAMA Cardiol 2017; 2: 1025-9.

136. Lam CS, Lyass A, Kraigher-Krainer E, et al. Cardiac dysfunction and noncardiac dysfunction as precursors of heart failure with reduced and preserved ejection fraction in the community. Circulation 2011; 124: 24-30.

137. Barr RG, Bluemke DA, Ahmed FS, et al. Percent emphysema, airflow obstruction, and impaired left ventricular filling. N Engl J Med 2010; 362: 217-27.

138. Yoshihisa A, Sato Y, Yokokawa T, et al. Liver fibrosis score predicts mortality in heart failure patients with preserved ejection fraction. ESC Heart Fail 2018; 5: 262-70.

139. Ather S, Chan W, Bozkurt B, et al. Impact of noncardiac comorbidities on morbidity and mortality in a predominantly male population with heart failure and preserved versus reduced ejection fraction. J Am Coll Cardiol 2012; 59: 998-1005.

140. Ter Maaten JM, Damman K, Verhaar MC, et al. Connecting heart failure with preserved ejection fraction and 
renal dysfunction: the role of endothelial dysfunction and inflammation. Eur J Heart Fail 2016; 18: 588-98.

141. Shah SJ, Aistrup GL, Gupta DK, et al. Ultrastructural and cellular basis for the development of abnormal myocardial mechanics during the transition from hypertension to heart failure. Am J Physiol Heart Circ Physiol 2014; 306: H88-100.

142. Ahmed A, Rich MW, Sanders PW, et al. Chronic kidney disease associated mortality in diastoli versus systolic heart failure: a propensity matched study. Am J Cardiol 2007; 99: 393-8.

143. Go AS, Yang J, Ackerson LM, et al. Hemoglobin level, chronic kidney disease, and the risks of death and hospitalization in adults with chronic heart failure: the Anemia in Chronic Heart Failure: Outcomes and Resource Utilization (ANCHOR) Study. Circulation 2006; 113: 2713-23.

144. Huerta A, López B, Ravassa S, et al. Association of cystatin $C$ with heart failure with preserved ejection fraction in elderly hypertensive patients: potential role of altered collagen metabolism. J Hypertens 2016; 34: 130-8.

145. Li X, Zhu H, Li P, et al. Serum cystatin C concentration as an independent marker for hypertensive left ventricular hypertrophy. J Geriatr Cardiol 2013; 10: 286-90.

146. Moran A, Katz R, Smith NL, et al. Cystatin C concentration as a predictor of systolic and diastolic heart failure. J Card Fail 2008; 14: 19-26.

147. Bielecka-Dabrowa A, Sakowicz A, Pietrucha T, et al. The profile of selected single nucleotide polymorphisms in patients with hypertension and heart failure with preserved and mid-range ejection fraction. Sci Rep 2017; 7: 8974.

148. Unger ED, Dubin RF, Deo R, et al. Association of chronic kidney disease with abnormal cardiac mechanics and adverse outcomes in patients with heart failure and preserved ejection fraction. Eur J Heart Fail 2016; 18: 103-12.

149. Gori M, Senni M, Gupta DK, et al.; PARAMOUNT Investigators. Association between renal function and cardiovascular structure and function in heart failure with preserved ejection fraction. Eur Heart J 2014; 35: 3442-51.

150. Lopez B, Gonzalez A, Hermida N, Laviades C Dıez J. Myocardial fibrosis in chronic kidney disease: potential benefits of torasemide. Kidney Int 2008; 74 (Suppl): S19-23.

151. Richards AM, Januzzi JL Jr, Troughton RW. Natriuretic peptides in heart failure with preserved ejection fraction. Heart Fail Clin 2014; 10: 453-70.

152. Zhu WH, Chen LY, Dai HL, Chen JH, Chen Y, Fang LZ. Correlation between $\mathrm{B}$ type natriuretic peptide and metabolic risk factors. Arch Med Sci 2016; 12: 334-40.

153. Alagiakrishnan K, Banach M, Jones LG, Datta S, Ahmed A, Aronow WS. Update on diastolic heart failure or heart failure with preserved ejection fraction in the older adults. Ann Med 2013; 45: 37-50.

154. Piechota M, Banach M, Jacoń A, Rysz J. Natriuretic peptides in cardiovascular diseases. Cell Mol Biol Lett 2008; 13: 155-81.

155. Yang H, Wu C, Xiao Y, Zhou S. Connexin and fibrosis related microRNAs in complex fractionated atrial electrograms. Arch Med Sci 2015; 11: 679-82.

156. Sucharov CC, Kao DP, Port JD, et al. Myocardial microRNAs associated with reverse remodeling in human heart failure. JCI Insight 2017; 2: e89169.

157. Vegter EL, van der Meer P, de Windt LJ, Pinto YM, Voors AA. MicroRNAs in heart failure: from biomarker to target for therapy. Eur J Heart Fail 2016; 18: 457-68.
158. Schmitter D, Voors AA, van der Harst P. HFpEF vs. HFrEF: can microRNAs advance the diagnosis? Eur J Heart Fail 2015; 17: 351-4. 